\title{
Effects of the Longitudinal Surface Roughness on Fiber Pull-Out Behavior in Carbon Fiber-Reinforced Epoxy Resin Composites
}

Yin Yao
Shaohua Chen
e-mail: chenshaohua72@hotmail.com
The State Key Laboratory of
Nonlinear Mechanics,
Institute of Mechanics,
Chinese Academy of Sciences,
Beijing, 100190, China

\section{Introduction}

Fiber-reinforced composites (FRC), which consist of fibers and matrix with plenty of interfaces between them, have a wide range of applications in various engineering artifacts, such as machine components, aerospace, and civil engineering structures, etc. [1]. In the composite system, both fibers and matrix maintain their physical and chemical identities and complement each other with their respective advantages. Therefore, FRC can be designed to have desirable properties (e.g., stiffness, strength, or toughness) for various engineering purposes, which cannot be achieved by any of the constituent materials acting alone [2].

Till now, many research have been focused on the mechanical properties and optimal design of FRC [3-5]. As a reinforcing phase, the fiber is expected to have significant strength and stiffness so that the load bearing capacity of composites can be effectively improved. Meanwhile, the weight reduction of the composite structure is also a critical factor that should be considered, especially for the aerospace and automotive utilizations. To meet the two aspects, carbon fiber is a very favorable choice, owing to its attractive characteristics of high stiffness, high strength, and light weight [6-9]. In many applications, carbon fibers are mixed into a thermosetting polymer matrix (e.g., epoxy

\footnotetext{
${ }^{1}$ Corresponding author.

Contributed by the Applied Mechanics Division of ASME for publication in the Journal of APPLIEd Mechanics. Manuscript received March 30, 2012; final manuscript received August 18, 2012; accepted manuscript posted August 23, 2012 published online January 22, 2013. Assoc. Editor: Daining Fang.
}

resin) with a good thermal stability and chemical resistance to produce carbon fiber-reinforced epoxy composite, which is a typical representative of advanced composites and widely used in aeronautical, marine, and automobile industries $[1,10,11]$.

As pointed out by many researchers, the performances of carbon/epoxy composites are largely dependent on the properties of fiber/matrix interface $[6,12-15]$. Strong interface adhesion guarantees efficient load transfer from the resin matrix to carbon fibers with high strength and stiffness so that the advantages of the reinforcements can be brought into full play. However, substantial studies have proved that carbon fibers have inert surfaces and always achieve weak adhesion to the organic resin matrix [16-18], leading to the occurrence of interfacial failure, such as interface debonding and fiber pull-out. Many surface modification techniques, such as oxidation, electrochemical, and plasma treatments, are developed in order to increase the chemical functions and roughness on the carbon fiber's surface; thus the chemical and physical interactions between carbon fiber and resin matrix can be strengthened. It is of great significance for the optimal design of carbon/epoxy composites with high qualities [19-22].

One of the surface modification techniques is to increase carbon fiber's surface roughness by etching treatment $[21,23,24]$. Using the scanning electron microscopy (SEM), it can be clearly observed that on the surfaces of treated carbon fibers, a large number of microridges and grooves distribute parallel in the fiber's longitudinal direction, leading to the longitudinal surface roughness [25-29]. As a result, the interfacial contact area is enlarged and the mechanical interlocking between fibers and matrix is enhanced. It has been proved by the fiber 
pull-out (FPO) tests, which show apparently increasing average interfacial shear strength (apparent IFSS) with the increase of longitudinal ridges and striations on carbon fiber's surface [26,28,29].

At present, the importance of carbon fiber's surface roughness in improving the interface adhesion is well recognized. Much attention has been paid to this issue, but most is confined to experimental investigations, in which fiber pull-out tests are commonly used to evaluate the enhancement of IFSS due to the surface roughness. Relevant theoretical studies are still very limited. In the early research on silicon-carbon ( $\mathrm{SiC})$ and metal fibers, Kerans and Parthasarathy [30], Liu et al. [31], Parthasarathy et al. [32], and Chai and Mai [33] have proposed a series of analytical models to explore the effects of interface roughness on the fiber pull-out and push-out behaviors, which provided several basic approaches to describe the rough surface topography (e.g., Fourier series, periodic trigonometric functions, and linear roughness profiles). Since many longitudinal striations are initially generated on carbon fiber's surface in the manufacturing process, longitudinal surface roughness is much easier to be produced than any other roughness patterns during surface modifications $[7,29]$. However, in the previous theoretical research, roughness of interface was generally assumed to be a circumferential one, i.e., the microridges and grooves distribute perpendicularly to the longitudinal direction of fibers, which is actually not consistent with the reality in experiments and practice $[27,28]$. The existing theoretical models did not reflect the realistic surface roughness of carbon fiber. A theoretical model considering the effect of longitudinal roughness of interface on the mechanical behaviors of carbon fiber-reinforced composites is still lacking, which should be more consistent with the experimental facts and has more instructive significance for practical designs of carbon/epoxy composites.

In this paper, such a theoretical model is established, in which longitudinal microridges and grooves on carbon fiber's surface are characterized and an improved shear-lag model is established. The influence mechanisms of longitudinal roughness on carbon fiber's pull-out from a thermosetting epoxy resin matrix are investigated, in which the surface roughness is evaluated by a ratio of the microscale amplitude to the wavelength of the roughness. Using this model, closed-form solutions to the axial stress of carbon fibers and the apparent IFSS are obtained, through which the effects of the longitudinal surface roughness on the fiber stress distribution and improvement of interfacial adhesions are discussed in detail. Comparisons between the theoretical predictions and the experimental results are also carried out.

\section{Theoretical Model and Analysis}

2.1 Characterization of the Longitudinal Surface Roughness. According to recent experiments [27-29], an idealized configuration of carbon fiber segment with a rough surface is sketched in Fig. 1. The longitudinal microridges and grooves on the fiber's surface are assumed to have a periodical distribution in the circumferential direction and the fiber's cross section keeps uniform in the axial direction. Consequently, the fiber's radius $r_{f}$ can be described by a cosine function of the central angle $\theta$, independent of the coordinate $z$, which is similar to Jiang et al. [34] and Waters et al. [35]:

$$
r_{f}(\theta)=r_{f 0}+\Delta \cos \frac{2 \pi r_{f 0} \theta}{\lambda}
$$

where $r_{f 0}$ represents the radius of carbon fiber with a smooth surface as shown in Fig. $1(c) . \Delta$ and $\lambda$ are the microscale amplitude and wavelength of the wavy cross section, respectively. Then the area and perimeter of the cross section can be obtained:

$$
\begin{aligned}
& A_{f}=\int_{0}^{2 \pi} d \theta \int_{0}^{r_{f}(\theta)} r d r \approx \pi r_{f 0}^{2} \\
& c_{f}=\oint_{s_{f}} d s=\int_{0}^{2 \pi} r_{f}(\theta) \sqrt{1+\frac{1}{\left[r_{f}(\theta)\right]^{2}}\left[\frac{d r_{f}(\theta)}{d \theta}\right]^{2}} d \theta
\end{aligned}
$$

Compared to the case with a smooth surface, the area of fiber's cross section keeps almost unchanged due to the small size of surface asperities, while the perimeter is enlarged, leading to an augmentation of the total surface area of the carbon fiber. It should be noted that when $\Delta \rightarrow 0$ or $\lambda \rightarrow \infty$, the carbon fiber's surface tends to be smooth and the perimeter $c_{f}$ will approach $2 \pi r_{f 0}$.

The special morphology of carbon fiber's surface with longitudinal roughness considered in the present paper has been analytically characterized by Eqs. (1) and (2), which will be incorporated into the classical shear-lag equation in the following text.

2.2 An Improved Shear-Lag Model With the Effect of Longitudinal Surface Roughness. Considering the carbon fiber's longitudinal surface roughness, an improved shear-lag model shown in Fig. 2 is established as compared to the classical one, in which a single fiber with longitudinal surface roughness is embedded in a cylindrical epoxy resin matrix. The carbon fiber has a radius $r_{f}$ and the radius of the cylindrical matrix is $r_{m} . L$ is the total length of the embedded carbon fiber. $\beta$ denotes the debonded fraction as shown by the dashed part in Fig. 2(a). Both the carbon fiber and resin matrix are assumed to be linear elastic and isotropic materials with $E_{f}, \nu_{f}, \kappa_{f}, E_{m}, \nu_{m}, \kappa_{m}$ being their Young's moduli, Poisson's ratios, and thermal expansion coefficients, respectively. The subscripts $f$ and $m$ denote the fiber and matrix, respectively. Similar to the pull-out test [36], a uniform tensile load $\sigma_{0}$ is applied to the fiber end (at $z=0$ ) and the lateral surface of matrix cylinder is stress-free. Then, the equilibrium condition between the externally and internally axial stresses requires

$$
\sigma_{0}=\sigma_{f}+\frac{1}{\gamma} \sigma_{m} ; \quad \gamma=\frac{A_{f}}{\pi r_{m}^{2}-A_{f}} \approx \frac{r_{f 0}^{2}}{r_{m}^{2}-r_{f 0}^{2}}
$$

in which $\sigma_{f}, \sigma_{m}$ are the axial stresses in the fiber and matrix, depending only on the coordinate $z$, i.e., $\sigma_{f}=\sigma_{f}(z), \sigma_{m}=\sigma_{m}(z)$ [37].

The stress-strain constitutive relations follow the general Hooke's law

$$
\begin{gathered}
\varepsilon_{\alpha r}=\frac{1}{E_{\alpha}}\left[\sigma_{\alpha r}-\nu_{\alpha}\left(\sigma_{\alpha \theta}+\sigma_{\alpha}\right)\right] \\
\varepsilon_{\alpha \theta}=\frac{1}{E_{\alpha}}\left[\sigma_{\alpha \theta}-\nu_{\alpha}\left(\sigma_{\alpha r}+\sigma_{\alpha}\right)\right] \quad(\alpha=f, m) \\
\varepsilon_{\alpha z}=\frac{1}{E_{\alpha}}\left[\sigma_{\alpha}-\nu_{\alpha}\left(\sigma_{\alpha r}+\sigma_{\alpha \theta}\right)\right] \quad\left(1+\nu_{m}\right) \\
\varepsilon_{f}^{r z}=\frac{2\left(1+\nu_{f}\right)}{E_{f}} \tau_{f}^{r z}, \quad \varepsilon_{m}^{r z}=\frac{2\left(1+\nu_{m}\right.}{E_{m}} \tau_{m}
\end{gathered}
$$

where $\sigma_{\alpha r}, \sigma_{\alpha \theta}, \sigma_{\alpha}, \tau_{\alpha}^{r z}$ are the radial, hoop, axial, and shear stresses, and $\varepsilon_{\alpha r}, \varepsilon_{\alpha \theta}, \varepsilon_{\alpha z}, \varepsilon_{\alpha}^{r z}$ are the corresponding strain components, respectively. Among them, the shear stress $\tau_{f}^{r z}$ and strain $\varepsilon_{f}^{r z}$ in the fiber are always neglected since the fiber mainly supports the axial tensile stress $[33,38]$. For an axisymmetric problem, it is known that the radial and hoop stresses in the fiber and matrix have the general forms as follows [39]:

$$
\sigma_{f r}=\sigma_{f \theta}=A, \quad \sigma_{m r}=\frac{B}{r^{2}}+C, \quad \sigma_{m \theta}=-\frac{B}{r^{2}}+C
$$

The unknown parameters $A, B, C$ in Eq. (5) are independent of the coordinate $r$, and their expressions can be found in Chai and Mai [33] and Gao et al. [37]:

$$
A=q_{0}-q_{\nu}, \quad B=\gamma r_{m}^{2}\left(q_{0}-q_{\nu}\right), \quad C=-\gamma\left(q_{0}-q_{\nu}\right)
$$

where $q_{0}$ and $q_{\nu}$ represent the interface radial stresses induced by the thermally residual stress and Poisson's effect, respectively, which can be expressed as 

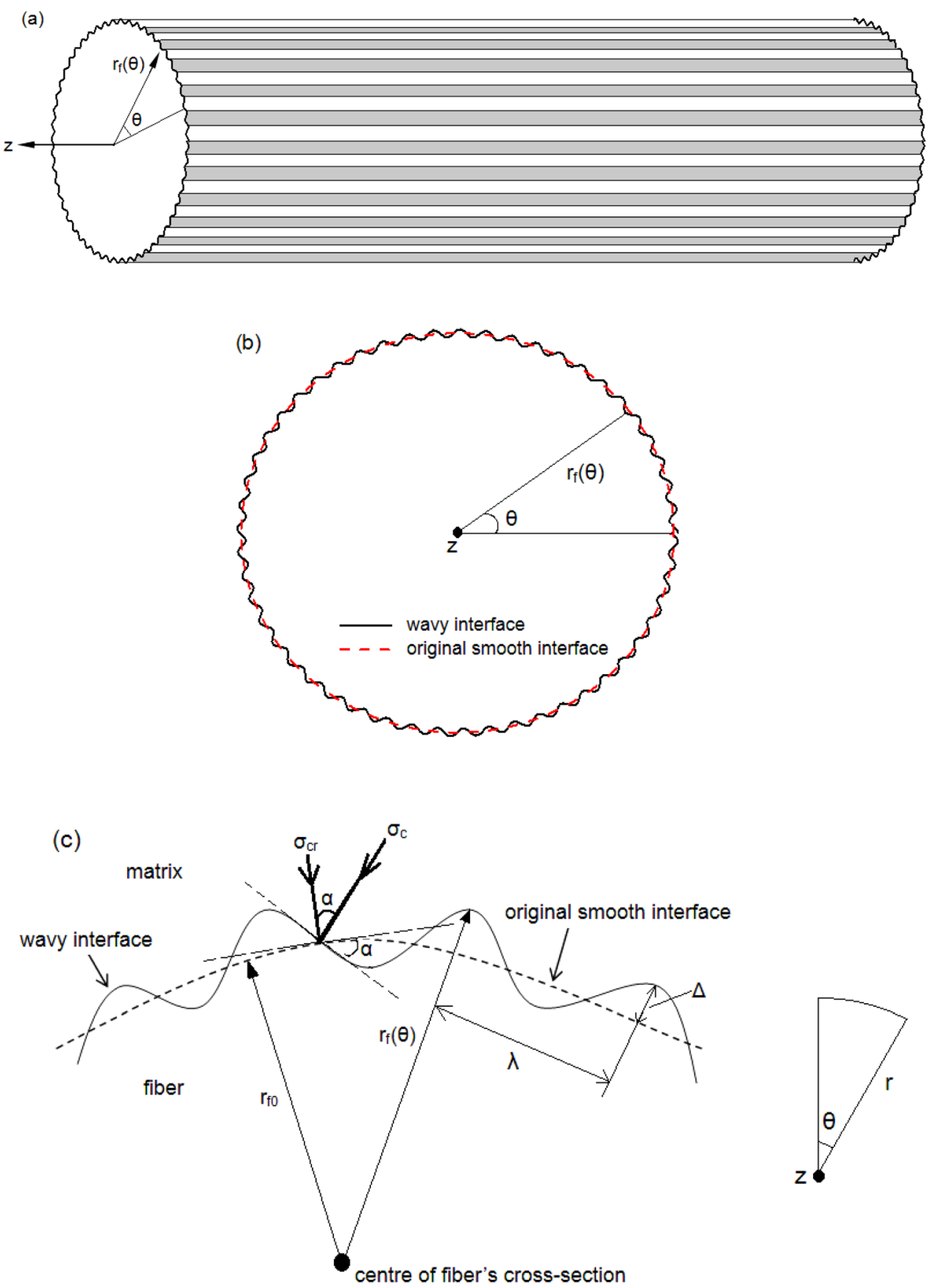

Fig. 1 Schematics of a carbon fiber with surface roughness. (a) 3D configuration of the fiber segment with longitudinal surface roughness; $(b)$ cross section of the carbon fiber with circumferentially wavy contour curve; (c) periodically wavy interface in the $r-\theta$ plane.

$$
\begin{aligned}
& q_{0}=\frac{E_{m}\left(\kappa_{m}-\kappa_{f}\right) \Delta T}{\alpha\left(1-\nu_{f}\right)+\left(1+2 \gamma+\nu_{m}\right)}, \quad q_{v}=k_{1} \sigma_{f}(z)-k_{2} \sigma_{0} \\
& \alpha=\frac{E_{m}}{E_{f}}, \quad k_{1}=\frac{\alpha \nu_{f}+\gamma \nu_{m}}{\alpha\left(1-\nu_{f}\right)+\left(1+2 \gamma+\nu_{m}\right)}, \\
& k_{2}=\frac{\gamma \nu_{m}}{\alpha\left(1-\nu_{f}\right)+\left(1+2 \gamma+\nu_{m}\right)}
\end{aligned}
$$

$\Delta T$ denotes the temperature difference during the curing process of carbon/epoxy composites [40]. The dimensionless parameters $k_{1}, k_{2}$ depend not only on the material constants of the fiber and matrix but also their sizes.

The equilibrium condition for an infinitesimal carbon fiber element with longitudinal surface roughness as shown in Fig. 2(b) yields

$$
\left(\sigma_{f}+d \sigma_{f}\right) A_{f}+d z \oint_{s_{f}} \tau_{i z} d s=\sigma_{f} A_{f}
$$

which is equivalent to

$$
\frac{d \sigma_{f}}{d z}=-\frac{\oint_{s_{f}} \tau_{i z} d s}{\pi r_{f 0}^{2}}
$$

Eq. (9) is a general form of the improved shear-lag equation including the effect of rough interface geometries, in which $\tau_{i z}$ is the interface shear stress in the $z$ direction, i.e., $\tau_{i z}=\left(\tau_{m}^{r z}\right)_{r=r_{f}}$, and $d z$ is the length of the fiber element. Based on the improved shear-lag relation in Eq. (9), a closed-form solution of carbon fiber stress including the effect of longitudinal roughness can be achieved as follows. 

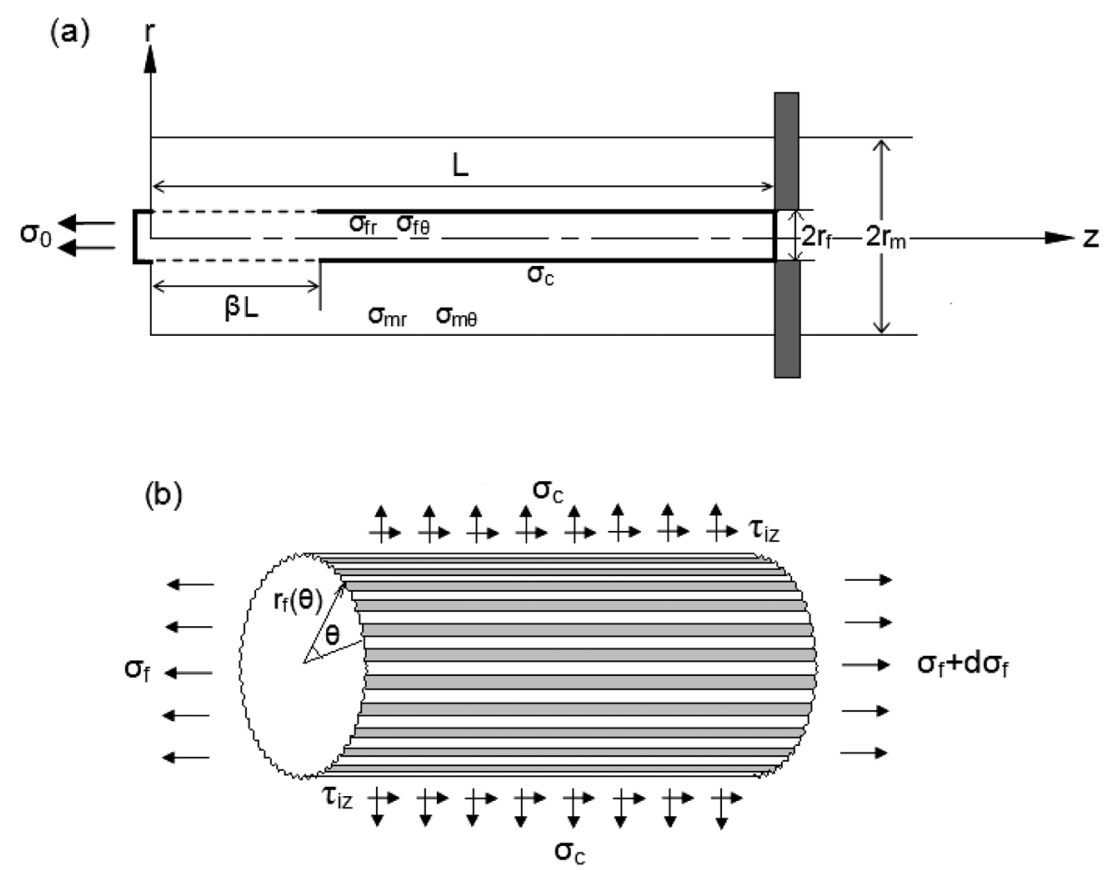

Fig. 2 Schematics of the loading form of carbon fiber-reinforced epoxy resin matrix composites. (a) Cylindrical model for a single fiber pulling out from the matrix; (b) an infinitesimal carbon fiber element with longitudinal surface roughness.

2.3 Closed-Form Solution of the Carbon Fiber Stress in the Debonded Region. The surface roughness considered in our paper is longitudinal not circumferential. The tangential direction of each point on the surface with longitudinal roughness is consistent with the direction of the interface shear stress. Coulomb's law is valid for such a wavy surface, which has been adopted even for the surface with circumferential roughness by Liu et al. [31] and Chai and Mai [33], so that the interface shear stress satisfies

$$
\tau_{i z}=-\mu \sigma_{c} \quad(0 \leq z \leq \beta L)
$$

where $\mu$ is the friction coefficient and the contact pressure $\sigma_{c}$ at each point is the component perpendicular to the tangential direction of the point, as shown in Fig. 1(c), which is the difference between cases of smooth surface and rough one. The decomposition of $\sigma_{c}$ in the curvilinear coordinate system yields

$$
\sigma_{c r}=\sigma_{c} \cos \alpha=\frac{\sigma_{c}}{\sqrt{1+\frac{1}{\left[r_{f}(\theta)\right]^{2}}\left[\frac{d r_{f}(\theta)}{d \theta}\right]^{2}}}
$$

where $\sigma_{c r}$ is the interface radial pressure normal to the original smooth interface. $\alpha$ is the angle between the tangential planes of the original smooth and rough interfaces as shown in Fig. 1(c). Apparently, the cosine of $\alpha$ is relevant to the rough interface geometries.

In the present model, it is assumed that no radial mismatch occurs between the fiber and matrix during pull-out due to the same direction of the longitudinal microridges and grooves as that of the frictional sliding. Once debonding happens, the relative sliding between the fiber and matrix is along a straight and smooth path in the present model, instead of a sawtooth or sinusoidalshaped path observed in the cases with a circumferential roughness $[30,32]$. Therefore, $\sigma_{c r}$ does not contain the additional radial stress induced by asperities misfits $[31,33,41]$. Meanwhile, since the microscale asperities on the rough interface are rather small as compared to the fiber's radius, $\sigma_{c r}$ has the following expression:

$$
\sigma_{c r}=\left(\sigma_{f r}\right)_{r=r_{f}}=q_{0}-q_{\nu}
$$

Combining Eqs. (2), and (9)-(12) yields

$$
\frac{d \sigma_{f}}{d z}=\frac{\mu\left(q_{0}-q_{v}\right)}{\pi r_{f 0}^{2}} \int_{0}^{2 \pi}\left\{1+\frac{1}{\left[r_{f}(\theta)\right]^{2}}\left[\frac{d r_{f}(\theta)}{d \theta}\right]^{2}\right\} r_{f}(\theta) d \theta
$$

which is the detailed improved shear-lag equation considering the longitudinal surface roughness in the present paper. When $\Delta \rightarrow 0$ or $\lambda \rightarrow \infty$, the integral in Eq. (13) tends to be $2 \pi r_{f 0}$. Equation (13) can then be naturally reduced to the classical one [37]:

$$
\frac{d \sigma_{f}}{d z}=\frac{2 \mu\left(q_{0}-q_{v}\right)}{r_{f 0}}
$$

Let $\bar{\Delta}=\Delta / r_{f 0}, \bar{\lambda}=\lambda / r_{f 0}, \bar{z}=z / L, \bar{r}_{f}=r_{f}(\theta) / r_{f 0}$. The aspect ratio can be denoted as $\rho=L / 2 r_{f} \approx L / 2 r_{f 0}$, then, Eq. (13) is rewritten as

$$
\frac{d \sigma_{f}}{d \bar{z}}=\frac{2 \rho \mu\left(q_{0}-q_{v}\right)}{\pi} \int_{0}^{2 \pi}\left\{1+\frac{1}{\left[\bar{r}_{f}(\theta)\right]^{2}} \frac{4 \pi^{2} \bar{\Delta}^{2}}{\bar{\lambda}^{2}} \sin \frac{2 \pi \theta}{\bar{\lambda}}\right\} \bar{r}_{f}(\theta) d \theta
$$

where $\bar{r}_{f}(\theta)=1+\bar{\Delta} \cos (2 \pi \theta / \bar{\lambda})$.

Based on Eqs. (7) and (15), a closed-form solution of the carbon fiber stress in the debonded region can be obtained as

$$
\begin{aligned}
\sigma_{f}(\bar{z})= & \frac{q_{0}+k_{2} \sigma_{0}}{k_{1}}-\left(\frac{q_{0}+k_{2} \sigma_{0}}{k_{1}}-\sigma_{0}\right) \\
& \times \exp \left(-\frac{2 r_{1} \rho \mu k_{1} \bar{z}}{\pi}\right) \quad(0 \leq \bar{z} \leq \beta)
\end{aligned}
$$

where $r_{1}=\int_{0}^{2 \pi}\left\{1+\frac{1}{\left[\bar{r}_{f}(\theta)\right]^{2}} \frac{4 \pi^{2} \bar{\Delta}^{2}}{\bar{\lambda}^{2}} \sin \frac{2 \pi \theta}{\lambda}\right\} \bar{r}_{f}(\theta) d \theta$.

2.4 Closed-Form Solution of the Carbon Fiber Stress in the Bonded Region. In the bonded region $(\beta \leq \bar{z} \leq 1)$, according to the equilibrium of axial forces of matrix annulus [42], we have 


$$
\left\{\begin{array}{c}
\frac{d \sigma_{m}}{d z}=\frac{\oint_{s_{f}} \tau_{i z} d s}{\pi r_{m}^{2}-A_{f}}=\frac{\oint_{s_{f}} \tau_{i z} d s}{\pi\left(r_{m}^{2}-r_{f 0}^{2}\right)} \\
\frac{d \sigma_{m}}{d z}=\frac{2 r}{r_{m}^{2}-r^{2}} \tau_{m}^{r z} \quad\left(r_{f}<r<r_{m}\right)
\end{array}\right.
$$

which yields

$$
\oint_{s_{f}} \tau_{i z} d s=\frac{2 \pi\left(r_{m}^{2}-r_{f 0}^{2}\right) r}{r_{m}^{2}-r^{2}} \tau_{m}^{r z}=\frac{2 \pi\left(r_{m}^{2}-r_{f 0}^{2}\right) r}{r_{m}^{2}-r^{2}} \frac{E_{m}}{2\left(1+\nu_{m}\right)} \frac{\partial w_{m z}}{\partial r}
$$

Here $w_{m z}$ is the axial displacement of the matrix, and $\tau_{i z}$ is independent of $r$. Integrating Eq. (18) with respect to $r$ leads to

$$
\begin{aligned}
\oint_{s_{f}} \tau_{i z} d s & =\frac{E_{m}}{1+\nu_{m}} \frac{\pi\left(r_{m}^{2}-r_{f 0}^{2}\right)}{r_{m}^{2} \ln \left(r_{m} / r_{f}\right)-\frac{r_{m}^{2}-r_{f}^{2}}{2}}\left[\left(w_{m z}\right)_{r=r_{m}}-\left(w_{m z}\right)_{r=r_{f}}\right] \\
& \approx \frac{E_{m}}{1+\nu_{m}} \frac{\pi\left(r_{m}^{2}-r_{f 0}^{2}\right)}{r_{m}^{2} \ln \left(r_{m} / r_{f 0}\right)-\frac{r_{m}^{2}-r_{f 0}^{2}}{2}}\left[\left(w_{m z}\right)_{r=r_{m}}-\left(w_{m z}\right)_{r=r_{f}}\right]
\end{aligned}
$$

Differentiating Eq. (19) with respect to $z$ and considering the nonslipping feature of the interface in the bonded region yield

$$
\frac{d\left(\oint_{s_{f}} \tau_{i z} d s\right)}{d z}=\frac{E_{m}}{1+\nu_{m}} \frac{\pi\left(r_{m}^{2}-r_{f 0}^{2}\right)}{r_{m}^{2} \ln \left(r_{m} / r_{f 0}\right)-\frac{r_{m}^{2}-r_{f 0}^{2}}{2}}\left[\left(\varepsilon_{m z}\right)_{r=r_{m}}-\left(\varepsilon_{f z}\right)_{r=r_{f}}\right]
$$

The shear-lag equation in the bonded region can then be obtained from Eqs. (4)-(7), (9), and (20):

$$
\frac{d^{2} \sigma_{f}}{d z^{2}}-\eta_{1} \sigma_{f}=-\eta_{1} \eta_{2} \sigma_{0}+\eta_{1} \eta_{3} q_{0}
$$

in which

$$
\begin{aligned}
& \eta_{1}=\frac{2}{1+\nu_{m}} \frac{(\alpha+\gamma)-2 k_{1}\left(\alpha \nu_{f}+\gamma \nu_{m}\right)}{2 \gamma r_{m}^{2} \ln \left(r_{m} / r_{f 0}\right)-r_{f 0}^{2}}, \\
& \eta_{2}=\frac{\gamma\left(1-2 k_{1} \nu_{m}\right)}{(\alpha+\gamma)-2 k_{1}\left(\alpha \nu_{f}+\gamma \nu_{m}\right)}, \\
& \eta_{3}=\frac{2\left(\alpha \nu_{f}+\gamma \nu_{m}\right)}{(\alpha+\gamma)-2 k_{1}\left(\alpha \nu_{f}+\gamma \nu_{m}\right)}
\end{aligned}
$$

where the two parameters $k_{1}, k_{2}$ are given in Eq. (7).

Let $\bar{z}=z / L, \bar{r}_{m}=r_{m} / r_{f 0}$, the dimensionless form of Eq. (21) can be written as

$$
\begin{aligned}
& \frac{d^{2} \sigma_{f}}{d \bar{z}^{2}}-4 \rho^{2} \bar{\eta}_{1} \sigma_{f}=-4 \rho^{2} \bar{\eta}_{1} \eta_{2} \sigma_{0}+4 \rho^{2} \bar{\eta}_{1} \eta_{3} q_{0} \\
& \bar{\eta}_{1}=\frac{2}{1+\nu_{m}} \frac{(\alpha+\gamma)-2 k_{1}\left(\alpha \nu_{f}+\gamma \nu_{m}\right)}{2 \gamma \bar{r}_{m}^{2} \ln \left(\bar{r}_{m}\right)-1}
\end{aligned}
$$

Consider the boundary conditions

$$
\begin{array}{ll}
\bar{z}=\beta: & \sigma_{f}=\sigma_{e} \\
\bar{z}=1: & \sigma_{f}=0
\end{array}
$$

where $\sigma_{e}$ denotes the carbon fiber stress at the dividing point between the debonded and bonded regions. With the help of Eq. (16), we have

$$
\sigma_{e}=\frac{q_{0}+k_{2} \sigma_{0}}{k_{1}}-\left(\frac{q_{0}+k_{2} \sigma_{0}}{k_{1}}-\sigma_{0}\right) \exp \left(-\frac{2 r_{1} \rho \mu k_{1} \beta}{\pi}\right)
$$

Then, the closed-form solution of the carbon fiber stress in the bonded region, based on Eqs. (23)-(25), can be obtained as

$$
\begin{aligned}
\sigma_{f}(\bar{z})= & \sigma_{e} \frac{\sinh \left[2 \rho \overline{\bar{\eta}}_{1}(1-\bar{z})\right]}{\sinh \left[2 \rho{\sqrt{\bar{\eta}_{1}}}_{1}(1-\beta)\right]}+\left(\eta_{2} \sigma_{0}-\eta_{3} q_{0}\right) \\
& \times\left(1-\frac{\sinh \left[2 \rho \sqrt{\bar{\eta}_{1}}(1-\bar{z})\right]+\sinh \left[2 \rho \sqrt{\bar{\eta}_{1}}(\bar{z}-\beta)\right]}{\sinh \left[2 \rho{\sqrt{\bar{\eta}_{1}}}_{1}(1-\beta)\right]}\right) \\
& \times(\beta \leq \bar{z} \leq 1)
\end{aligned}
$$

2.5 Relation Between the Tensile Load and the CarbonFiber's Displacement During Pull-Out. The whole pull-out process can be generally divided into two stages, i.e., the interface debonding and fiber pull-out [43], in which the required tensile load and fiber's sliding displacement are expressed as $\sigma_{0 d}, \delta_{d}$ and $\sigma_{0 p}, \delta_{p}$, respectively. The subscript "d" denotes "debonding" and the one "p" represents "pull-out."

2.5.1 Partial Interface Debonding. Based on the Griffith energy balance equation [33], the interface debonding criterion can be derived as

$$
G_{c r}=\frac{\partial U_{t}}{\partial S_{d}}=\frac{\partial U_{t}}{\partial\left(\beta L \oint_{s_{f}} d s\right)}=\frac{1}{L \oint_{s_{f}} d s} \frac{\partial U_{t}}{\partial \beta}
$$

where $G_{c r}$ is the interface fracture toughness, $S_{d}$ denotes the debonded area. $U_{t}$ is the total elastic strain energy of the carbon/ epoxy composite:

$$
\begin{aligned}
U_{t}= & U_{f t}+U_{m t} \\
= & \frac{1}{2 E_{f}} \int_{0}^{L} \int_{0}^{r_{f}}\left[\sigma_{f}^{2}+\sigma_{f r}^{2}+\sigma_{f \theta}^{2}-2 \nu_{f}\left(\sigma_{f} \sigma_{f r}+\sigma_{f} \sigma_{f \theta}+\sigma_{f r} \sigma_{f \theta}\right)\right] 2 \pi r d r d z \\
& +\frac{1}{2 E_{m}} \int_{0}^{L} \int_{r_{f}}^{r_{m}}\left[\sigma_{m}^{2}+\sigma_{m r}^{2}+\sigma_{m \theta}^{2}-2 \nu_{m}\left(\sigma_{m} \sigma_{m r}+\sigma_{m} \sigma_{m \theta}+\sigma_{m r} \sigma_{m \theta}\right)\right. \\
& \left.+2\left(1+\nu_{m}\right)\left(\tau_{m}^{r z}\right)^{2}\right] 2 \pi r d r d z
\end{aligned}
$$

where $U_{f t}$ and $U_{m t}$ refer to the elastic strain energy in the carbon fiber and epoxy resin matrix, respectively. All the stress components in Eq. (28) are provided in Eqs. (3), (5)-(7), (16), (17), and (26). Then, Eq. (27) can be rewritten as a quadratic function of $\sigma_{0 d}$ :

$$
G_{c r} L \oint_{s_{f}} d s=\frac{\partial U_{f t}}{\partial \beta}+\frac{\partial U_{m t}}{\partial \beta}=w_{1} \sigma_{0 d}^{2}+w_{2} \sigma_{0 d}+w_{3}
$$

where $w_{1} \sim w_{3}$ are given by Eqs. (A18) and (A19) in the Appendix as well as the details of derivation of Eq. (29). Solving Eq. (29) and abandoning the negative root result in

$$
\sigma_{0 d}=\frac{1}{2 w_{1}}\left[-w_{2}+\sqrt{w_{2}^{2}-4 w_{1}\left(w_{3}-G_{c r} L \oint_{s_{f}} d s\right)}\right]
$$

where the tensile load $\sigma_{0 d}$ is a function of the debonded fraction $\beta$, i.e., $\sigma_{0 d}=\sigma_{0 d}(\beta)$. 
The relative displacement $v(z)$ of carbon fiber in the debonded region satisfies

$$
\frac{d v(z)}{d z}=\frac{d}{d z}\left|w_{f z}-w_{m z}\right|=\left|\varepsilon_{f z}-\varepsilon_{m z}\right|, \quad(0 \leq z \leq \beta L)
$$

where $w_{f z}$ and $w_{m z}$ denote the axial displacements of the fiber and matrix. The axial strain components $\varepsilon_{f z}$ and $\varepsilon_{m z}$ in the fiber and matrix can be found from Eqs. (3)-(7). In addition, we have $v(z=\beta L)=0$. Then, $v(z)$ can be determined from Eq. (31) as

$$
\bar{v}(\bar{z})=\frac{v(z)}{L}=\frac{1}{E_{m}}\left|\lambda_{1} f_{1}(\bar{z})+\left(\lambda_{2} \sigma_{0 d}+\lambda_{3} q_{0}\right)(\beta-\bar{z})\right|
$$

where

$$
\begin{aligned}
\lambda_{1}=\alpha+\gamma-2 k_{1}\left(\alpha \nu_{f}+\gamma \nu_{m}\right), & \lambda_{2}=2 k_{2}\left(\alpha \nu_{f}+\gamma \nu_{m}\right)-\gamma \\
\lambda_{3}= & 2\left(\alpha \nu_{f}+\gamma \nu_{m}\right), \quad \bar{z}=z / L \\
f_{1}= & \frac{\left(q_{0}+k_{2} \sigma_{0 d}\right)(\beta-\bar{z})}{k_{1}} \\
& -\frac{\pi\left[q_{0}+\left(k_{2}-k_{1}\right) \sigma_{0 d}\right]}{2 k_{1}^{2} r_{1} \mu \rho}\left(e^{-\frac{2 \rho k_{1} r_{1} \mu \bar{z}}{\pi}}-e^{-\frac{2 \rho k_{1} r_{1} \mu \beta}{\pi}}\right)
\end{aligned}
$$

Parameters $k_{1}, k_{2}, \alpha, \gamma, r_{1}$ are provided in Eqs. (3), (7), and (16). The dimensionless carbon fiber's displacement $\bar{\delta}_{d}$ at $\bar{z}=0$ can be achieved as

$$
\begin{aligned}
& \bar{\delta}_{d}=\bar{v}(0)=\frac{1}{E_{m}}\left|\lambda_{1} f_{1}(0)+\beta\left(\lambda_{2} \sigma_{0 d}+\lambda_{3} q_{0}\right)\right| \\
& f_{1}(0)=\frac{\beta\left(q_{0}+k_{2} \sigma_{0 d}\right)}{k_{1}}-\frac{\pi\left[q_{0}+\left(k_{2}-k_{1}\right) \sigma_{0 d}\right]}{2 k_{1}^{2} r_{1} \mu \rho} \quad\left(1-e^{-\frac{2 \rho k_{1} r_{1} \mu \beta}{\pi}}\right)
\end{aligned}
$$

The relation between the tensile load $\sigma_{0 d}$ and the carbon fiber's sliding displacement $\bar{\delta}_{d}$ in the debonding stage is now explicitly expressed by Eqs. (30) and (34), where both quantities are functions of parameter $\beta$ :

$$
\left\{\begin{array}{l}
\sigma_{0 d}=\sigma_{0 d}(\beta) \\
\bar{\delta}_{d}=\bar{\delta}_{d}(\beta)
\end{array}\right.
$$

2.5.2 The Pull-Out Stage. When the interface is completely debonded, i.e., $\beta=1$, the carbon fiber can be pulled out from the epoxy resin matrix. At this stage, the stress transferring along the whole interface is assumed to abide by the Coulomb's frictional law $[43,44]$. The normalized pull-out displacement $\bar{\delta}_{p}$ ranges from $\left(\bar{\delta}_{d}\right)_{\beta=1}$ to 1 . The normalized fiber length embedded in the matrix is defined as $l_{e}$, which equals $1-\bar{\delta}_{p}$.

Combining Eqs. (10)-(15) and using the boundary condition $\left(\sigma_{f}\right)_{\bar{z}=l_{e}}=0$ yield the relation between the tensile load $\sigma_{0 p}$ and the displacement $\bar{\delta}_{p}$,

$$
\sigma_{0 p}=\frac{\left[1-e^{\frac{2 \rho k_{1} r_{1} \mu\left(1-\bar{\delta}_{p}\right)}{\pi}}\right] q_{0}}{k_{1}-k_{2}+k_{2} e^{\frac{2 \rho k_{1} r_{1} \mu\left(1-\delta_{p}\right)}{\pi}}}, \quad\left(\left(\bar{\delta}_{d}\right)_{\beta=1} \leq \bar{\delta}_{p} \leq 1\right)
$$

where the parameter $r_{1}$ is given in Eq. (16) characterizing the effect of surface roughness. It can be found that when $\bar{\delta}_{p}$ changes from $\left(\bar{\delta}_{d}\right)_{\beta=1}$ to 1 , the corresponding tensile load $\sigma_{0 p}$ will decrease from $\left(\sigma_{0 d}\right)_{\beta=1}$ to zero.

2.5.3 Apparent IFSS. An apparent IFSS $\tau_{a}$ is introduced in this section to evaluate the interfacial adhesion. It was often used in fiber pull-out tests $[28,45,46]$ and defined as the average shear stress over the whole interface region when the tensile load reaches the maximum and catastrophic interface failure tends to occur.

$$
\tau_{a}=\frac{\sigma_{0}^{\max } \pi r_{f 0}^{2}}{2 \pi r_{f 0} L}=\frac{\sigma_{0}^{\max }}{4 \rho}
$$

where $\sigma_{0}^{\max }$ is the maximum tensile load occurring in the debonding stage.

One can see that the apparent IFSS, which reflects the load bearing capacity of the fiber/matrix interface in a composite system, depends on not only the carbon fiber's geometry but also the maximum tensile load. The apparent IFSS will be discussed in the present model of carbon fiber-reinforced epoxy composites with longitudinal interface roughness in the following text.

\section{Results and Discussions}

According to Huang and Young [47], stress transfer at a perfect interface between the carbon fiber and epoxy resin matrix should attribute to the elastic shear deformation, because the epoxy resin is a thermosetting polymer. According to Hughes [6], Zhang et al. [40], and Piggott [48], the parameters of the carbon fiber and epoxy resin matrix are taken as $E_{f}=230 \mathrm{GPa}, E_{m}=3 \mathrm{GPa}$, $\nu_{f}=0.2, \quad \nu_{m}=0.35, \quad \kappa_{f}=12 \times 10^{-6} /{ }^{0} \mathrm{C}, \quad \kappa_{m}=55 \times 10^{-6} /{ }^{0} \mathrm{C}$, $\mu=0.3, G_{c r}=200 \mathrm{~J} / \mathrm{m}^{2}$. The nondimensional radius of the matrix is adopted as $\bar{r}_{m}=r_{m} / r_{f 0}=4$, the aspect ratio of the carbon fiber is $\rho=L / 2 r_{f 0}=10$, and the temperature difference $\Delta T=-150^{\circ} \mathrm{C}$, unless otherwise stated.

The interface roughness is evaluated by the ratio of the amplitude to the wavelength $\Delta / \lambda$. When $\Delta / \lambda$ approaches zero, the fiber/matrix interface tends to be smooth. According to the experimental results [28], the values of $\Delta / \lambda$ change with the etching time as shown in Table 1. One can see that the amplitude increases, while the wavelength decreases with an increasing treating time. In other words, the longitudinal grooves become deeper and the number of grooves increases, leading to a reducing wavelength of the roughness. In contrast to the micrometer radius of carbon fibers, the amplitude of asperities is very small, which has a nanoscale.

3.1 The Tensile Load in the Debonding and Pull-Out Stages. Figure 3 shows the relation between the normalized tensile load $\bar{\sigma}_{0 d}\left(\bar{\sigma}_{0 d}=\sigma_{0 d} / E_{m}\right)$ in the debonding stage and the debonded fraction $\beta$ for different interface roughness. From Fig. 3, one can see that the tensile load initially increases from a finite value, which is very similar to the analytical results in Chai and Mai [33] and Piggort [48]. The initially finite value corresponds to the initiation of interface debonding, i.e., $\beta=0$. When the tensile load exceeds a peak value, catastrophic interface failure occurs [46], the remaining bonded interface debonds very rapidly, and the tensile load drops sharply in an unstable fashion. There is a critical value of the debonded fraction $\beta$, at which the tensile load $\bar{\sigma}_{0}$ achieves the maximum. We call the critical value $\beta_{c}$, which can be theoretically determined from Eq. (35). Numerical calculation on $d \sigma_{0 d} / d \beta=0$ shows $\beta_{c} \approx 0.63$. It is interesting to find that the critical value $\beta_{c}$ is

Table 1 Values of $\Delta / \lambda$ for different etching time (carbon fiber radius $\left.r_{f 0}=3.5 \mu \mathrm{m}\right)$

\begin{tabular}{llll}
\hline \hline Treating time (hour) & \multicolumn{1}{c}{$\Delta / r_{f 0}$} & $\lambda / r_{f 0}$ & $\Delta / \lambda$ \\
\hline $0 \mathrm{~h}$ & 0 & $-\overline{-}$ & 0 \\
$24 \mathrm{~h}$ & 0.0055 & 0.286 & 0.019 \\
$48 \mathrm{~h}$ & 0.0072 & 0.171 & 0.042 \\
$72 \mathrm{~h}$ & 0.009 & 0.122 & 0.074 \\
$96 \mathrm{~h}$ & 0.012 & 0.107 & 0.112 \\
$120 \mathrm{~h}$ & 0.016 & 0.086 & 0.186 \\
\hline \hline
\end{tabular}




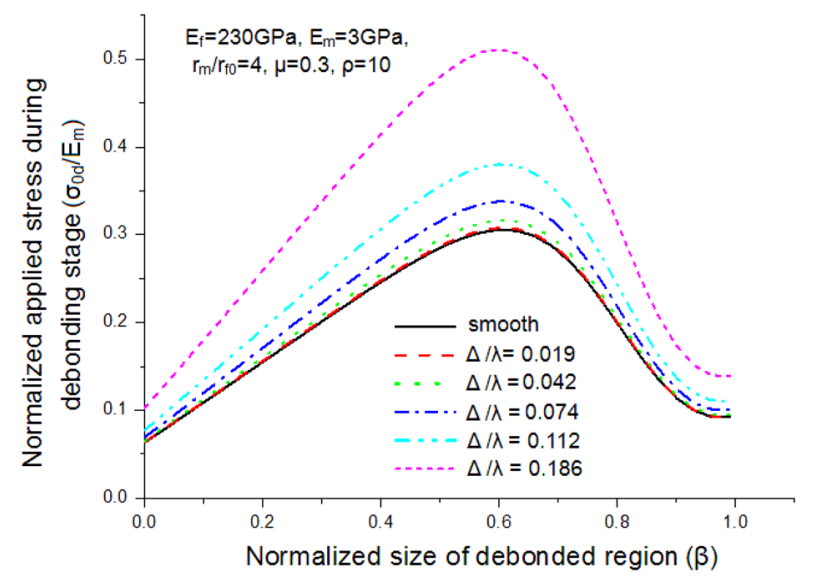

Fig. 3 The normalized tensile load during the debonding stage versus the debonded fraction for different interface roughness

almost insensitive to the interface roughness and coincides with that predicted by Piggort [48] for carbon/epoxy composites with smooth interfaces, while the tensile load increases with an increasing interface roughness $\Delta / \lambda$ for a fixed debonded fraction. It means that the rougher the carbon fiber's surface, the higher tensile load is needed to cause the interface debonding.

The relation of the tensile load versus the displacement for the whole pull-out process is plotted in Fig. 4(a). The experimental result from Bismarck et al. [49] for carbon/epoxy composites is also presented for comparison. One can see that the theoretical prediction has the same order magnitude as the experimental one. The magnitude difference between the theoretical and experimental results may be primarily caused by several aspects, such as the values of the material parameters, the surface roughness profiles, geometry of the pull-out device, the mechanical and thermal properties of the carbon fiber and epoxy resin matrix, etc. Two main features of carbon fiber pull-out behavior are captured theoretically, which was also exhibited by the existing experiment results and the theoretical ones for the case of smooth interfaces: (1) Fig. 4(a) shows that the tensile load increases rapidly from zero to its peak value with a very small displacement. It means that the interface debonding stage occupies a small part of the whole pull-out process in carbon/epoxy composites [50], which is consistent with the experimental observation [49]; (2) Fig. 4(b), as an amplification of the theoretical results in Fig. 4(a), clearly depicts the transition from the debonding stage to the fiber pull-out stage, in which point $A$ corresponds to the turning point at which $\beta=\beta_{c}$ and the tensile load begins to decrease, while point $B$ corresponds to the complete debonding with $\beta=1$. From point $A$ to point $B$, the tensile load drops precipitously from its maximum to a finite value, with a very small increment of fiber's displacement, which exhibits an instable "friction-controlled" pattern as described in Gao et al. [37] and Yue and Cheung [51]. The stress transfer at the debonded interface is governed by sliding friction and the catastrophic interface debonding occurs immediately after the tensile load reaches its maximum. All the features are consistent with the experimental results shown in Fig. 4(c) [49].

3.2 Relations Among the Carbon Fiber Stress, Interface Roughness and Debonded Fraction. The relation between the debonded fraction $\beta$ and the interface roughness $\Delta / \lambda$ under a fixed tensile load $\bar{\sigma}_{0}$ is shown in Fig. 5(a) and the one between the tensile load $\bar{\sigma}_{0}$ and the interface roughness $\Delta / \lambda$ for a fixed $\beta$ is shown in Fig. 5(b). It is found that under a given tensile load, an increasing interface roughness $\Delta / \lambda$ leads to an obvious reduction of the length of the debonded region, which suggests that the longitudinal surface roughness on carbon fiber's surface can effectively suppress the development of interfacial failure. On the other hand, when the interface becomes rougher, a larger tensile load is
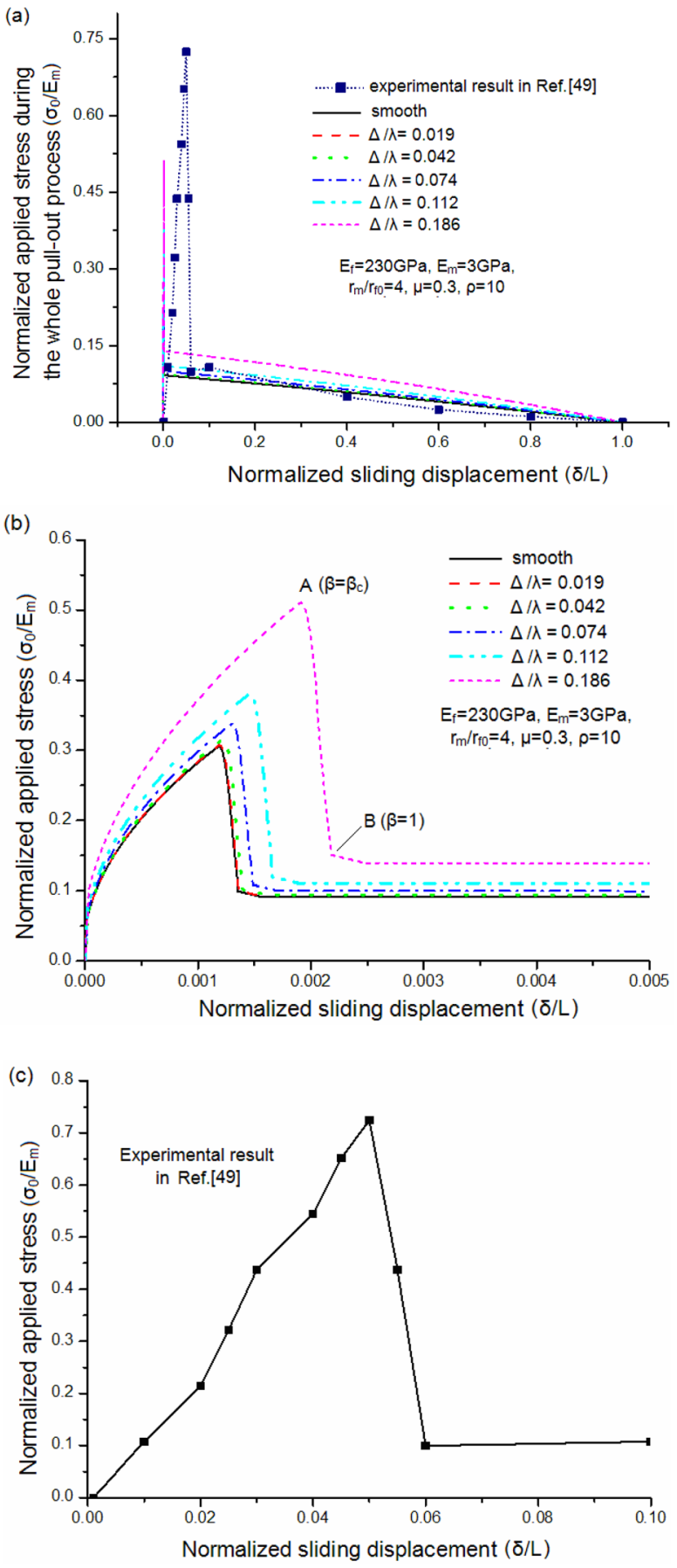

Fig. 4 The whole pull-out process of a carbon fiber-reinforced epoxy resin matrix composite. (a) The relation between the normalized tensile load and the normalized sliding displacement for different interface roughness; (b) amplification of the curves of the tensile load versus sliding displacement in the region of $0 \leq \bar{\delta} \leq 0.005$; (c) amplification of the experimental results [49] of load-displacement curve in the region of $0 \leq \bar{\delta} \leq 0.1$.

required to maintain the same size of the interface debonding region.

Under a fixed tensile load, the distribution of carbon fiber stress in the debonded region is plotted in Fig. 6(a). When the interface becomes rougher, the fiber stress decreases due to an increasing interface frictional resistance, which is qualitatively consistent with the numerical results in Liu and Kagawa [52]. While Liu and Kagawa [52] characterized the interface roughness by a frictional coefficient in their numerical simulations, the microscale geometric 

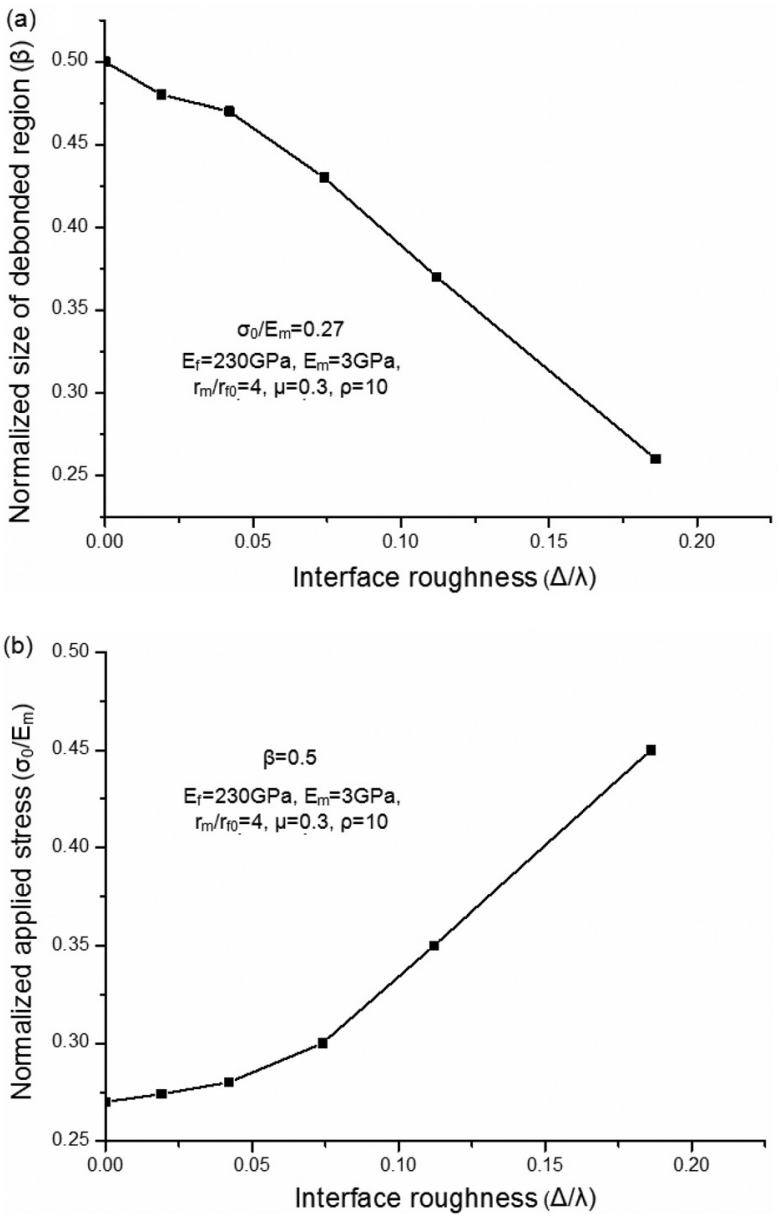

Fig. 5 The effects of the interface roughness on the mechanical behaviors of composites. (a) The debonded fraction $\beta$ as a function of the roughness ratio $\Delta / \lambda$ under a fixed tensile load; (b) the tensile load $\bar{\sigma}_{0}$ varying with the roughness ratio $\Delta / \lambda$ for a given debonded fraction.

parameters of the interface roughness are denoted as $\Delta$ and $\lambda$ in the present paper, which should be more realistic.

The results in Fig. 6(a) should be very useful for the research on crack bridging mechanisms in carbon fiber-reinforced epoxy composites. As pointed out by Marshall et al. [53] and Hsueh [54], a macroscopic matrix crack can be bridged by aligned fibers, which debond from and slip frictionally against the matrix. If the axial stress carried by the bridging fiber is too large and causes the fiber to break at weak points, the overall toughness and strength of composites will be weakened consequently. Therefore, when a bridging crack occurs in carbon/epoxy composites, it is necessary to ensure the safety of fibers. Inspired by the results in Fig. 6(a), the stress level in the bridging fibers can be reduced by properly roughening the interface. However, a large interface roughness should also be avoided because a strong interfacial adhesion would make the interface difficult to debond and matrix cracks are very likely to propagate catastrophically [55]. Therefore, the problem of achieving an optimal interface roughness to meet improved toughness and high strength is put forward. This important issue is out of the scope of this paper and will be investigated in the future.

Figure $6(b)$ gives the distributions of carbon fiber stress along the whole fiber length for a fixed debonded fraction. The tensile load increases with an increasing roughness, which naturally leads to a larger axial stress in the carbon fiber.

3.3 The Apparent IFSS With the Effect of Interface Roughness. Using the experimental data in Table 1, we plot the variations of the apparent IFSS versus the surface etching time in (a)
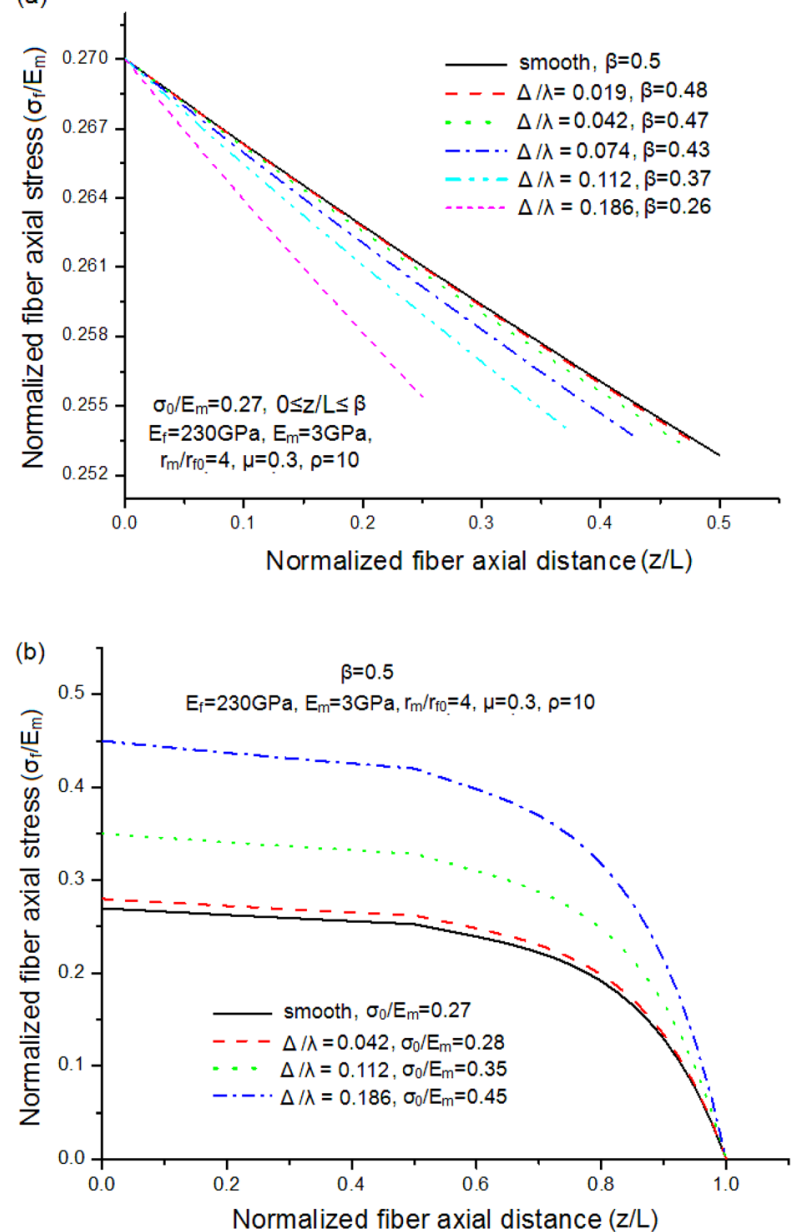

Fig. 6 The distributions of carbon fiber axial stress along the fiber length. (a) In the debonded region under a given tensile load; $(b)$ along the whole fiber length with a fixed debonded fraction.

Fig. 7, where the experimental results [28] are also presented for comparison. It is found that the apparent IFSS predicted by the present theoretical model has the same order magnitude as the experimental one and increases with the increasing treating time, reflecting a positive effect of the interface roughness. The

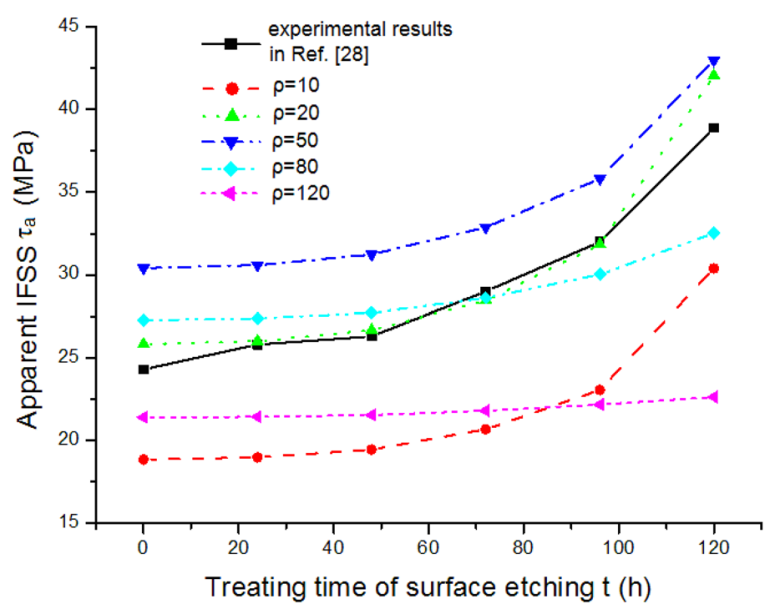

Fig. 7 The effect of the etching time on the apparent IFSS for cases with different aspect ratios, where the experimental results [28] are shown for comparison 


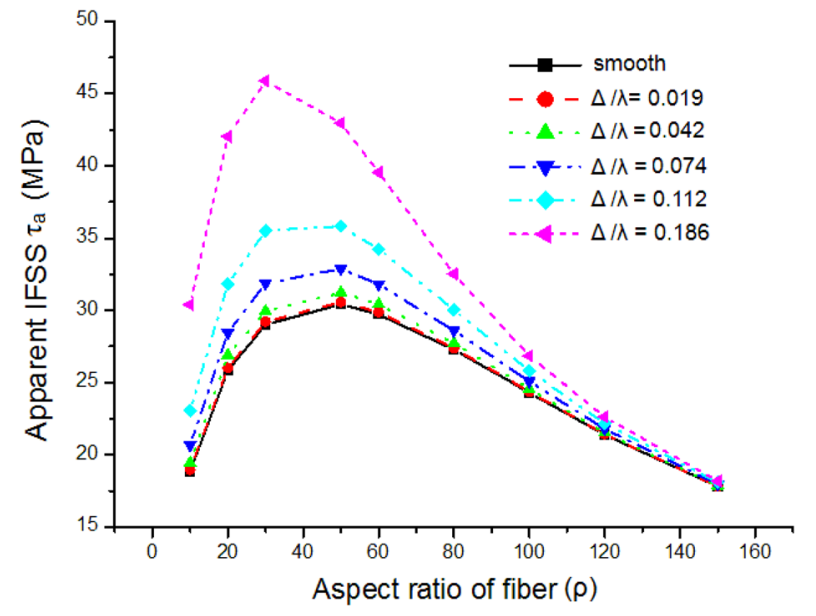

Fig. 8 The relation of the apparent IFSS versus the carbon fiber's aspect ratio for different interface roughness

theoretical result in the case with aspect ratio $\rho=20$ is almost identical with the experimental one [28], which also verifies the physical reasonableness of our theoretical model. It is worth noting that, for a given aspect ratio of carbon fibers, the apparent IFSS increases initially and then decreases with the increasing aspect ratio. Furthermore, the larger the aspect ratio, the less effect of the surface roughness on the apparent IFSS is. A much clearer result is shown in Fig. 8, where the apparent IFSS varies as a function of the aspect ratio of carbon fibers. It is clearly found that, for a determined interface roughness, there always exists an optimal aspect ratio of carbon fibers, at which the apparent IFSS reaches the maximum. Even in the case with a smooth interface, an optimal aspect ratio still exists to render the apparent IFSS achieving the maximum. Obviously, the maximum value increases with the increasing interface roughness.

However, it is difficult to find the optimal aspect ratio of carbon fibers analytically due to a transcendental equation of $\rho$ derived from $d \tau_{a} / d \rho=0$. From the results in Figs. 7 and 8, one can infer that the optimal aspect ratio of carbon fibers is about 30-45, which should be useful for the design of strong interfaces in carbon/epoxy composites.

\section{Conclusions}

According to the realistic surface morphology of carbon fibers in fiber-reinforced composites, the surface roughness of carbon fibers is described as longitudinally periodical microscale ridges and grooves. A more realistic theoretical model for carbon fiberreinforced epoxy resin matrix composites is established in the present paper, in which the effects of surface roughness of carbon fibers on the mechanical features of the fiber/matrix interface are considered. An improved shear-lag model is established and closed-form solutions of the axial stress of carbon fibers and the apparent IFSS are obtained, through which the effects of the longitudinal surface roughness on the distribution of fiber stress and improvement of interfacial adhesion are discussed in detail. It is found that the interface strength can be enhanced by the interface roughness. Under a fixed external tensile load, both the axial stress in carbon fibers and the size of the debonded region can be reduced due to the interface roughness. The load-displacement curves of the whole pull-out process predicted by the present analytical model are qualitatively consistent with the experimental ones. In addition, the apparent IFSS considering the effect of interface roughness is evaluated, which agrees well with the existing experimental results. Furthermore, it is interesting to find that an optimal aspect ratio of carbon fibers exists, at which the apparent interface shear strength achieves the maximum and its enhancement due to the longitudinal surface roughness is the most significant. It is inferred that the optimal aspect ratio of carbon fibers is about $30-45$. The theoretical results in the present paper should have instructive significance for practical designs of carbon fiber-reinforced epoxy composites.

\section{Acknowledgment}

The work reported here is supported by National Science Foundation of China through Grants \#10972220, \#11125211, \#11021262 and the Key Project \#2012CB937500.

\section{Appendix}

From Eq. (28), we have

$$
\begin{aligned}
U_{f t}= & \frac{1}{2 E_{f}} \int_{0}^{L} \int_{0}^{r_{f}}\left[\sigma_{f}^{2}+\sigma_{f r}^{2}+\sigma_{f \theta}^{2}-2 \nu_{f}\left(\sigma_{f} \sigma_{f r}+\sigma_{f} \sigma_{f \theta}+\sigma_{f r} \sigma_{f \theta}\right)\right] 2 \pi r d r d z \\
U_{m t}= & \frac{1}{2 E_{m}} \int_{0}^{L} \int_{r_{f}}^{r_{m}}\left[\sigma_{m}^{2}+\sigma_{m r}^{2}+\sigma_{m \theta}^{2}-2 \nu_{m}\right. \\
& \left.\times\left(\sigma_{m} \sigma_{m r}+\sigma_{m} \sigma_{m \theta}+\sigma_{m r} \sigma_{m \theta}\right)+2\left(1+\nu_{m}\right)\left(\tau_{m}^{r z}\right)^{2}\right] 2 \pi r d r d z
\end{aligned}
$$

where $U_{f t}$ and $U_{m t}$ refer to the elastic strain energies in the fiber and matrix, respectively. According to Eqs. (3), (5)-(7), and (17), $U_{f t}, U_{m t}$ can be reformulated as

$$
\begin{aligned}
U_{f t}= & \frac{\pi r_{f 0}^{2} L}{2 E_{f}} \int_{0}^{1}\left(h_{1} \sigma_{f}^{2}+h_{2} \sigma_{f}+h_{3}\right) d \bar{z} \\
U_{m t}= & \frac{\pi r_{f 0}^{2} L}{2 E_{m}} \int_{0}^{1}\left(h_{4} \sigma_{f}^{2}+h_{5} \sigma_{f}+h_{6}\right) d \bar{z} \\
& +\frac{\pi r_{f 0}^{3}\left(1+\nu_{m}\right) a_{6}}{E_{m} \rho} \int_{0}^{1}\left(-\gamma \frac{d \sigma_{f}}{d \bar{z}}\right)^{2} d \bar{z}
\end{aligned}
$$

in which

$$
\begin{aligned}
h_{1}= & 1+2\left(1-\nu_{f}\right) k_{1}^{2}+4 k_{1} \nu_{f}, h_{2}=-4\left(q_{0}+k_{2} \sigma_{0}\right)\left[k_{1}\left(1-\nu_{f}\right)+\nu_{f}\right] \\
h_{3}= & 2\left(1-\nu_{f}\right)\left(q_{0}+k_{2} \sigma_{0}\right)^{2} \\
h_{4}= & \gamma^{2}\left[\pi r_{f 0}^{2}+k_{1}^{2}\left(a_{1}+a_{2}\right)\right]-2 \nu_{m} k_{1} \gamma\left(a_{3}-a_{4}-a_{5} \gamma k_{1}\right) \\
h_{5}= & -2 \gamma^{2}\left[\pi r_{f 0}^{2} \sigma_{0}+k_{1}\left(a_{1}+a_{2}+2 \nu_{m} a_{5}\right)\left(q_{0}+k_{2} \sigma_{0}\right)\right] \\
& +2 \nu_{m} \gamma\left(a_{3}-a_{4}\right)\left[q_{0}+\left(k_{1}+k_{2}\right) \sigma_{0}\right] \\
h_{6}= & \gamma^{2}\left[\pi r_{f 0}^{2} \sigma_{0}^{2}+\left(a_{1}+a_{2}+2 \nu_{m} a_{5}\right)\left(q_{0}+k_{2} \sigma_{0}\right)^{2}\right] \\
- & 2 \nu_{m} \gamma \sigma_{0}\left(a_{3}-a_{4}\right)\left(q_{0}+k_{2} \sigma_{0}\right) \\
a_{1}= & \frac{1}{2}\left[-1+\bar{r}_{m}^{4}-\bar{r}_{m}^{2} \ln \left(\bar{r}_{m}\right)\right], a_{2}=\frac{1}{2}\left[-1+\bar{r}_{m}^{4}+\bar{r}_{m}^{2} \ln \left(\bar{r}_{m}\right)\right] \\
a_{3}= & \bar{r}_{m}^{2} \ln \left(\bar{r}_{m}\right)-\frac{\bar{r}_{m}^{2}-1}{2}, a_{4}=\bar{r}_{m}^{2} \ln \left(\bar{r}_{m}\right)+\frac{\bar{r}_{m}^{2}-1}{2} \\
a_{5}= & \frac{\left(\bar{r}_{m}^{2}-1\right)^{2}}{2}, a_{6}=\bar{r}_{m}^{4} \ln \left(\bar{r}_{m}\right)-\frac{1}{4}+\bar{r}_{m}^{2}-\frac{3 \bar{r}_{m}^{4}}{4} \\
&
\end{aligned}
$$

Differentiating Eq. (A2) with respect to $\beta$ yields

$$
\begin{aligned}
\frac{\partial U_{f t}}{\partial \beta}= & \frac{\pi r_{f 0}^{2} L}{2 E_{f}} \frac{\partial}{\partial \beta}\left[\int_{0}^{1}\left(h_{1} \sigma_{f}^{2}+h_{2} \sigma_{f}\right) d \bar{z}\right] \\
\frac{\partial U_{m t}}{\partial \beta}= & \frac{\pi r_{f 0}^{2} L}{2 E_{m}} \frac{\partial}{\partial \beta}\left[\int_{0}^{1}\left(h_{4} \sigma_{f}^{2}+h_{5} \sigma_{f}\right) d \bar{z}\right] \\
& +\frac{\pi r_{f 0}^{3}\left(1+\nu_{m}\right) a_{6}}{E_{m} \rho} \frac{\partial}{\partial \beta}\left[\int_{0}^{1}\left(-\gamma \frac{d \sigma_{f}}{d \bar{z}}\right)^{2} d \bar{z}\right]
\end{aligned}
$$


The integrals in Eq. (A4) can be divided into several parts as follows:

$$
\begin{aligned}
& \frac{\partial}{\partial \beta}\left(\int_{0}^{1} h_{1} \sigma_{f}^{2} d \bar{z}\right)=h_{1} \frac{\partial}{\partial \beta}\left(\int_{0}^{\beta} \sigma_{f}^{2} d \bar{z}+\int_{\beta}^{1} \sigma_{f}^{2} d \bar{z}\right) \\
& \frac{\partial}{\partial \beta}\left(\int_{0}^{1} h_{2} \sigma_{f} d \bar{z}\right)=h_{2} \frac{\partial}{\partial \beta}\left(\int_{0}^{\beta} \sigma_{f} d \bar{z}+\int_{\beta}^{1} \sigma_{f} d \bar{z}\right) \\
& \frac{\partial}{\partial \beta}\left[\int_{0}^{1}\left(-\gamma \frac{d \sigma_{f}}{d \bar{z}}\right)^{2} d \bar{z}\right]=\gamma^{2} \frac{\partial}{\partial \beta}\left[\int_{0}^{\beta}\left(\frac{d \sigma_{f}}{d \bar{z}}\right)^{2} d \bar{z}+\int_{\beta}^{1}\left(\frac{d \sigma_{f}}{d \bar{z}}\right)^{2} d \bar{z}\right]
\end{aligned}
$$

With the help of Eqs. (16) and (27), the integrals in Eq. (A5) can be written as

$\frac{\partial}{\partial \beta} \int_{0}^{\beta} \sigma_{f}^{2} d \bar{z}=c_{1} \sigma_{0}^{2}+c_{2} \sigma_{0}+c_{3}, \quad \frac{\partial}{\partial \beta} \int_{\beta}^{1} \sigma_{f}^{2} d \bar{z}=c_{4} \sigma_{0}^{2}+c_{5} \sigma_{0}+c_{6}$,

$\frac{\partial}{\partial \beta} \int_{0}^{\beta} \sigma_{f} d \bar{z}=c_{7} \sigma_{0}+c_{8}, \quad \frac{\partial}{\partial \beta} \int_{\beta}^{1} \sigma_{f} d \bar{z}=c_{9} \sigma_{0}+c_{10}$

$\frac{\partial}{\partial \beta} \int_{0}^{\beta}\left(\frac{d \sigma_{f}}{d \bar{z}}\right)^{2} d \bar{z}=c_{11} \sigma_{0}^{2}+c_{12} \sigma_{0}+c_{13}$,

$\frac{\partial}{\partial \beta} \int_{\beta}^{1}\left(\frac{d \sigma_{f}}{d \bar{z}}\right)^{2} d \bar{z}=c_{14} \sigma_{0}^{2}+c_{15} \sigma_{0}+c_{16}$

where the coefficients $c_{1} \sim c_{3}$ and $c_{7} \sim c_{10}$ are determined as follows:

$$
c_{1}=\frac{e^{-\frac{4 \beta \rho k_{1} r_{1} \mu}{\pi}}\left[k_{1}+\left(e^{\frac{2 \beta \rho k_{1} r_{1} \mu}{\pi}}-1\right) k_{2}\right]^{2}}{k_{1}^{2}}
$$$$
c_{2}=\frac{1}{k_{1}^{2}}\left[2 q_{0}\left(e^{-\frac{2 \beta \rho k_{1} r_{1} \mu}{\pi}}-e^{-\frac{4 \beta \rho k_{1} r_{1} \mu}{\pi}}\right)\left(k_{1}-k_{2}+k_{2} e^{\frac{2 \beta \rho k_{1} r_{1} \mu}{\pi}}\right)\right]
$$$$
c_{3}=\frac{e^{-\frac{4 \beta \rho k_{1} r_{1} \mu}{\pi}}\left(e^{\frac{2 \beta \rho k_{1} r_{1} \mu}{\pi}}-1\right)^{2} q_{0}^{2}}{k_{1}^{2}}
$$$$
c_{7}=1-\frac{e^{-\frac{2 \beta \rho k_{1} r_{1} \mu}{\pi}}\left(1-e^{\frac{2 \beta \rho k_{1} r_{1} \mu}{\pi}}\right)\left(k_{2}-k_{1}\right)}{k_{1}},
$$$$
c_{8}=-\frac{e^{-\frac{2 \beta \rho k_{1} r_{1} \mu}{\pi}}\left(1-e^{\frac{2 \beta \rho k_{1} r_{1} \mu}{\pi}}\right) q_{0}}{k_{1}}
$$$$
c_{9}=-\frac{1}{2 k_{1} \pi \sqrt{\eta_{1}}}\left\{\frac{\pi \sqrt{\eta_{1}} e^{-\frac{2 \beta \rho k_{1} r_{1} \mu}{\pi}}}{\cosh ^{2}\left[\sqrt{\eta_{1}} \rho(\beta-1)\right]}\right.
$$$$
\times\left[\left(k_{1}-k_{2}\left(1-e^{\frac{2 \beta \rho k_{1} r_{1} \mu}{\pi}}\right)-k_{1} \eta_{2} e^{\frac{2 \beta \rho k_{1} r_{1} \mu}{\pi}}\right)\right]
$$$$
+e^{\frac{2 \beta \rho k_{1} r_{1} \mu}{\pi}} k_{1} \pi \sqrt{\eta_{1}} \eta_{2} \cosh \left[2 \sqrt{\eta_{1}} \rho(\beta-1)\right]
$$$$
\left.-k_{1} r_{1} \mu\left(k_{1}-k_{2}\right) \sinh \left[2 \sqrt{\eta_{1}} \rho(\beta-1)\right]\right\}
$$

$$
\begin{aligned}
c_{10}= & \frac{q_{0}}{2}\left\{-\frac{1-e^{-\frac{2 \beta \rho k_{1} r_{1} \mu}{\pi}}+2 k_{1} \eta_{3}}{k_{1} \cosh ^{2}\left[\sqrt{\eta_{1}} \rho(\beta-1)\right]}\right. \\
& \left.+2\left[\eta_{3}+\frac{r_{1} \mu e^{-\frac{2 \beta \rho k_{1} r_{1} \mu}{\pi}} \tanh \left[\sqrt{\eta_{1}} \rho(1-\beta)\right]}{\pi \sqrt{\eta_{1}}}\right]\right\}
\end{aligned}
$$

and the coefficients $c_{4} \sim c_{6}$ are

$$
\begin{aligned}
c_{4}= & \frac{e^{-\frac{4 \beta \rho k_{1} r_{1} \mu}{\pi}}}{4 k_{1}^{2} \sqrt{\eta_{1}} \pi \sinh ^{3}\left[2 \sqrt{\eta_{1}} \rho(1-\beta)\right]} d_{1} \\
& +\frac{e^{-\frac{2 \beta \rho k_{1} r_{1} \mu}{\pi}}}{4 k_{1}^{2} \sqrt{\eta_{1}} \pi \sinh ^{3}\left[2 \sqrt{\eta_{1}} \rho(1-\beta)\right]} d_{2} \\
& +\frac{d_{3}}{4 k_{1}^{2} \sqrt{\eta_{1}} \pi \sinh ^{3}\left[2 \sqrt{\eta_{1}} \rho(1-\beta)\right]}
\end{aligned}
$$

$$
c_{5}=-\frac{q_{0} e^{-\frac{4 \beta \rho k_{1} r_{1} \mu}{\pi}}}{4 k_{1}^{2} \sqrt{\eta_{1}} \pi \sinh ^{3}\left[2 \sqrt{\eta_{1}} \rho(1-\beta)\right]} d_{4}
$$

$$
-\frac{q_{0} e^{-\frac{2 \beta \rho k_{1} r_{1} \mu}{\pi}}}{4 k_{1}^{2} \sqrt{\eta_{1}} \pi \sinh ^{3}\left[2 \sqrt{\eta_{1}} \rho(1-\beta)\right]} d_{5}
$$

$$
-\frac{q_{0} d_{6}}{4 k_{1}^{2} \sqrt{\eta_{1}} \pi \sinh ^{3}\left[2 \sqrt{\eta_{1}} \rho(1-\beta)\right]}
$$

$$
c_{6}=-\frac{q_{0}^{2} e^{-\frac{4 \beta \rho k_{1} r_{1} \mu}{\pi}}}{4 k_{1}^{2} \sqrt{\eta_{1}} \pi \sinh ^{3}\left[2 \sqrt{\eta_{1}} \rho(1-\beta)\right]} d_{7}
$$

$$
\begin{aligned}
& -\frac{q_{0}^{2} e^{-\frac{2 \beta \rho k_{1} r_{1} \mu}{\pi}}}{4 k_{1}^{2} \sqrt{\eta_{1}} \pi \sinh ^{3}\left[2 \sqrt{\eta_{1}} \rho(1-\beta)\right]} d_{8} \\
& -\frac{q_{0}^{2} d_{9}}{4 k_{1}^{2} \sqrt{\eta_{1}} \pi \sinh ^{3}\left[2 \sqrt{\eta_{1}} \rho(1-\beta)\right]}
\end{aligned}
$$

The parameters $d_{1} \sim d_{9}$ in Eq. (A8) are

$$
\begin{aligned}
& d_{1}=\left(k_{1}-k_{2}\right)^{2}\left\{\left[k_{1} r_{1} \mu+8(\beta-1) \pi \eta_{1} \rho\right] \cosh \left[2 \sqrt{\eta_{1}} \rho(\beta-1)\right]\right. \\
& -k_{1} r_{1} \mu \cosh \left[6 \sqrt{\eta_{1}} \rho(\beta-1)\right]-4 \sqrt{\eta_{1}}\left[\pi-2 k_{1} r_{1} \mu \rho(\beta-1)\right] \\
& \left.\times \sinh \left[2 \sqrt{\eta_{1}} \rho(\beta-1)\right]\right\}, \\
& d_{2}=8\left(k_{2}-k_{1}\right)\left[k_{2} \pi \sqrt{\eta_{1}}+k_{1} k_{2} \mu \rho \sqrt{\eta_{1}} r_{1}(1-\beta)\right. \\
& \left.-k_{1}^{2} \mu \rho \sqrt{\eta_{1}} \eta_{2} r_{1}(1-\beta)\right] \sinh \left[2 \sqrt{\eta_{1}} \rho(1-\beta)\right] \\
& +\left(k_{2}-k_{1}\right)\left\{2 k_{1}\left(k_{1} r_{1} \eta_{2} \mu+6 \pi \eta_{1} \eta_{2} \rho-6 \beta \pi \eta_{1} \eta_{2} \rho\right)\right. \\
& -\left[k_{1}^{2} r_{1} \eta_{2} \mu+16 k_{2} \pi \eta_{1} \rho(\beta-1)+k_{1} k_{2} r_{1} \mu\right. \\
& \left.\left.-16 k_{1} \pi \eta_{1} \eta_{2} \rho(\beta-1)\right]\right\} \cosh \left[2 \sqrt{\eta_{1}} \rho(\beta-1)\right] \\
& -2 k_{1} \eta_{2}\left[k_{1} r_{1} \mu+2 \pi \eta_{1} \rho(\beta-1)\right] \cosh \left[4 \sqrt{\eta_{1}} \rho(\beta-1)\right] \\
& +k_{1} r_{1} \mu\left(k_{2}+\eta_{2}\right) \cosh \left[6 \sqrt{\eta_{1}} \rho(\beta-1)\right] \\
& +4 k_{1}^{2} r_{1} \mu \rho \sqrt{\eta_{1}} \eta_{2}(1-\beta) \sinh \left[4 \sqrt{\eta_{1}} \rho(1-\beta)\right], \\
& d_{3}=2 \pi \sqrt{\eta_{1}}\left\{k_{2}-k_{1} \eta_{2}+k_{1} \eta_{2} \cosh \left[2 \sqrt{\eta_{1}} \rho(\beta-1)\right]\right\} \\
& \times\left\{4(\beta-1)\left(k_{2}-k_{1} \eta_{2}\right) \sqrt{\eta_{1}} \rho \cosh \left[2 \sqrt{\eta_{1}} \rho(\beta-1)\right]\right\} \\
& -2\left(k_{2}+k_{1} \eta_{2}\right) \sinh \left[2 \sqrt{\eta_{1}} \rho(1-\beta)\right] \\
& +k_{1} \eta_{2}\left\{4(\beta-1) \sqrt{\eta_{1}} \rho+\sinh \left[4 \sqrt{\eta_{1}} \rho(1-\beta)\right]\right\}
\end{aligned}
$$

021015-10 / Vol. 80, MARCH 2013 


$$
\begin{aligned}
& d_{4}=-2\left(k_{1}-k_{2}\right)\left\{-\left[k_{1} r_{1} \mu+8(\beta-1) \pi \eta_{1} \rho\right] \cosh \left[2 \sqrt{\eta_{1}} \rho(\beta-1)\right]\right\} \\
& \left.+k_{1} r_{1} \mu \cosh \left[6 \sqrt{\eta_{1}} \rho(\beta-1)\right]+4 \sqrt{\eta_{1}}\left[\pi-2 k_{1} r_{1} \mu \rho(\beta-1)\right] \sinh \left[2 \sqrt{\eta_{1}} \rho(\beta-1)\right]\right\}, \\
& d_{5}=-2 k_{1}\left[\eta_{2}+\left(k_{1}-k_{2}\right) \eta_{3}\right]\left[k_{1} r_{1} \mu-6(\beta-1) \pi \eta_{1} \rho\right]+\left\{k_{1}^{2} \eta_{3} r_{1} \mu+32 k_{2}(\beta-1) \pi \eta_{1} \rho+k_{1}^{2}\left[r_{1} \mu\left(\eta_{2}-1-k_{2} \eta_{3}\right)\right.\right. \\
& \left.\left.-16(\beta-1) \pi \eta_{1} \eta_{3} \rho\right]+2 k_{1} \times\left[-8 \pi \eta_{1} \rho(\beta-1)\left(1+\eta_{2}\right)+k_{2} r_{1} \mu+8 k_{2} \pi \eta_{1} \eta_{3} \rho(\beta-1)\right]\right\} \cosh \left[2 \sqrt{\eta_{1}} \rho(\beta-1)\right] \\
& +2 k_{1}\left[\eta_{2}+\left(k_{1}-k_{2}\right) \eta_{3}\right]\left[k_{1} r_{1} \mu+2(\beta-1) \pi \eta_{1} \rho\right] \cosh \left[4 \sqrt{\eta_{1}} \rho(\beta-1)\right] \\
& +k_{1} r_{1} \mu\left(k_{1}-2 k_{2}-k_{1} \eta_{2}-k_{1}^{2} \eta_{3}+k_{1} k_{2} \eta_{3}\right) \cosh \left[6 \sqrt{\eta_{1}} \rho(\beta-1)\right] \\
& +8 \sqrt{\eta_{1}}\left[-2 k_{2} \pi-k_{1}^{3} r_{1} \mu \eta_{3} \rho(\beta-1)-k_{1}^{2} r_{1} \mu \rho(\beta-1)\left(\eta_{2}+1-k_{2} \eta_{3}\right)\right. \\
& \left.+k_{1} \pi+2 k_{1} k_{2} r_{1} \mu \rho(\beta-1)\right] \sinh \left[2 \sqrt{\eta_{1}} \rho(\beta-1)\right]+4 k_{1}^{2} r_{1} \mu \rho \sqrt{\eta_{1}}(\beta-1)\left[\eta_{2}+\left(k_{1}-k_{2}\right) \eta_{3}\right] \sinh \left[4 \sqrt{\eta_{1}} \rho(\beta-1)\right] \\
& d_{6}=12 k_{1} \pi \eta_{1} \rho(\beta-1)\left(\eta_{2}-k_{2} \eta_{3}+2 k_{1} \eta_{2} \eta_{3}\right)+2 \pi \sqrt{\eta_{1}}\left(4 k_{2}+5 k_{1}^{2} \eta_{2} \eta_{3}\right) \sinh \left[2 \sqrt{\eta_{1}} \rho(\beta-1)\right] \\
& -8 k_{1}^{2} \pi \sqrt{\eta_{1}} \eta_{2} \eta_{3} \sinh \left[4 \sqrt{\eta_{1}} \rho(\beta-1)\right]-16 \pi \eta_{1} \rho(\beta-1)\left[k_{2}+k_{1} k_{2} \eta_{3}-k_{1} \eta_{3}\left(1+2 k_{1} \eta_{3}\right)\right] \cosh \left[2 \sqrt{\eta_{1}} \rho(\beta-1)\right] \\
& -2 k_{1} \pi \eta_{1} \rho(\beta-1)\left(\eta_{2}-k_{2} \eta_{3}+2 k_{1} \eta_{2} \eta_{3}\right) \cosh \left[4 \sqrt{\eta_{1}} \rho(\beta-1)\right]+k_{1}^{2} \pi \sqrt{\eta_{1}} \eta_{2} \eta_{3} \sinh \left[6 \sqrt{\eta_{1}} \rho(\beta-1)\right] \\
& d_{7}=-\left[k_{1} r_{1} \mu+8(\beta-1) \pi \eta_{1} \rho\right] \cosh \left[2 \sqrt{\eta_{1}} \rho(\beta-1)\right]+k_{1} r_{1} \mu \cosh \left[6 \sqrt{\eta_{1}} \rho(\beta-1)\right] \\
& +4 \sqrt{\eta_{1}}\left[\pi-2 k_{1} r_{1} \mu \rho(\beta-1)\right] \sinh \left[2 \sqrt{\eta_{1}} \rho(\beta-1)\right] \\
& d_{8}=2 k_{1} \eta_{3}\left[k_{1} r_{1} \mu-6 \pi \eta_{1} \rho(\beta-1)\right]+\left[16 \pi \eta_{1} \rho(\beta-1)-k_{1}^{2} r_{1} \eta_{3} \mu+k_{1} r_{1} \mu+16(\beta-1) k_{1} \pi \eta_{1} \eta_{3} \rho\right] \cosh \left[2 \sqrt{\eta_{1}} \rho(\beta-1)\right] \\
& -2 k_{1} \eta_{3}\left[k_{1} r_{1} \mu+2 \pi \eta_{1} \rho(\beta-1)\right] \cosh \left[4 \sqrt{\eta_{1}} \rho(\beta-1)\right]+k_{1} r_{1} \mu\left(k_{1} \eta_{3}-1\right) \cosh \left[6 \sqrt{\eta_{1}} \rho(\beta-1)\right] \\
& -8 \sqrt{\eta_{1}}\left[\pi-k_{1} r_{1} \mu \rho(\beta-1)\left(1+k_{1} \eta_{3}\right)\right] \sinh \left[2 \sqrt{\eta_{1}} \rho(\beta-1)\right]-4 k_{1}^{2} r_{1} \sqrt{\eta_{1}} \eta_{3} \mu \rho(\beta-1) \sinh \left[4 \sqrt{\eta_{1}} \rho(\beta-1)\right] \\
& d_{9}=-2 \pi \sqrt{\eta_{1}}\left\{-1-k_{1} \eta_{3}+k_{1} \eta_{3} \cosh \left[2 \sqrt{\eta_{1}} \rho(\beta-1)\right]\right\}\left\{-4 \sqrt{\eta_{1}}(\beta-1)\left(1+k_{1} \eta_{3}\right) \cosh \left[2 \sqrt{\eta_{1}} \rho(\beta-1)\right]\right. \\
& \left.+2\left(1-k_{1} \eta_{3}\right) \sinh \left[2 \sqrt{\eta_{1}} \rho(\beta-1)\right]+4 k_{1} \eta_{3} \rho \sqrt{\eta_{1}}(\beta-1)+k_{1} \eta_{3} \sinh \left[4 \sqrt{\eta_{1}} \rho(\beta-1)\right]\right\}
\end{aligned}
$$

The coefficients $c_{11} \sim c_{16}$ and the parameters $d_{10} \sim d_{18}$ in Eq. (A13) are given as

$$
\begin{aligned}
& c_{11}=\frac{4 e^{-\frac{4 \beta \rho k_{1} r_{1} \mu}{\pi}}\left(k_{2}-k_{1}\right)^{2} r_{1}^{2} \mu^{2} \rho^{2}}{\pi^{2}}, \\
& c_{12}=\frac{8 e^{-\frac{4 \beta \rho k_{1} r_{1} \mu}{\pi}}\left(k_{2}-k_{1}\right) q_{0} r_{1}^{2} \mu^{2} \rho^{2}}{\pi^{2}}, \\
& c_{13}=\frac{4 e^{-\frac{4 \beta \rho k_{1} r_{1} \mu}{\pi}} q_{0}^{2} r_{1}^{2} \mu^{2} \rho^{2}}{\pi^{2}} \\
& c_{14}=\frac{\sqrt{\eta_{1}} \rho^{2} e^{-\frac{4 \beta \rho k_{1} r_{1} \mu}{\pi}}}{k_{1}^{2} \pi \sinh ^{3}\left[2 \sqrt{\eta_{1}} \rho(1-\beta)\right]} d_{10} \\
& +\frac{\sqrt{\eta_{1}} \rho^{2} e^{-\frac{2 \beta \rho k_{1} r_{1} \mu}{\pi}}}{k_{1}^{2} \pi \sinh ^{3}\left[2 \sqrt{\eta_{1}} \rho(1-\beta)\right]} d_{11}+\frac{\sqrt{\eta_{1}} \rho^{2} d_{12}}{k_{1}^{2} \pi \sinh ^{3}\left[2 \sqrt{\eta_{1}} \rho(1-\beta)\right]} \\
& c_{15}=\frac{\sqrt{\eta_{1}} \rho^{2} q_{0} e^{-\frac{4 \beta \rho k_{1} r_{1} \mu}{\pi}}}{k_{1}^{2} \pi \sinh ^{3}\left[2 \sqrt{\eta_{1}} \rho(1-\beta)\right]} d_{13} \\
& +\frac{\sqrt{\eta_{1}} \rho^{2} q_{0} e^{-\frac{2 \beta \rho k_{1} r_{1} \mu}{\pi}}}{k_{1}^{2} \pi \sinh ^{3}\left[2 \sqrt{\eta_{1}} \rho(1-\beta)\right]} d_{14}+\frac{\sqrt{\eta_{1}} \rho^{2} q_{0} d_{15}}{k_{1}^{2} \pi \sinh ^{3}\left[2 \sqrt{\eta_{1}} \rho(1-\beta)\right]} \\
& c_{16}=\frac{\sqrt{\eta_{1}} \rho^{2} q_{0}^{2} e^{-\frac{4 \beta \rho k_{1} r_{1} \mu}{\pi}}}{k_{1}^{2} \pi \sinh ^{3}\left[2 \sqrt{\eta_{1}} \rho(1-\beta)\right]} d_{16} \\
& +\frac{\sqrt{\eta_{1}} \rho^{2} q_{0}^{2} e^{-\frac{2 \beta \rho k_{1} r_{1} \mu}{\pi}}}{k_{1}^{2} \pi \sinh ^{3}\left[2 \sqrt{\eta_{1}} \rho(1-\beta)\right]} d_{17}+\frac{q_{0}^{2} d_{18}}{k_{1}^{2} \pi \sinh ^{3}\left[2 \sqrt{\eta_{1}} \rho(1-\beta)\right]}
\end{aligned}
$$

$$
\begin{aligned}
d_{10}= & \left(k_{1}-k_{2}\right)^{2}\left\{\left[k_{1} r_{1} \mu-8(\beta-1) \pi \eta_{1} \rho\right] \cosh \left[2 \sqrt{\eta_{1}} \rho(\beta-1)\right]\right. \\
& -k_{1} r_{1} \mu \cosh \left[6 \sqrt{\eta_{1}} \rho(\beta-1)\right]+8 \sqrt{\eta_{1}} \rho(\beta-1) \\
& \left.\times \sinh \left[2 \sqrt{\eta_{1}} \rho(\beta-1)\right]\right\} \\
d_{11}= & \left(k_{1}-k_{2}\right)\left\{\left(k_{2}-k_{1} \eta_{2}\right)\left[k_{1} r_{1} \mu-16(\beta-1) \pi \eta_{1} \rho\right]\right. \\
& \times \cosh \left[2 \sqrt{\eta_{1}} \rho(\beta-1)\right]-2 k_{1} \eta_{2}\left[k_{1} r_{1} \mu+2(\beta-1) \pi \eta_{1} \rho\right] \\
& \times \cosh \left[4 \sqrt{\eta_{1}} \rho(\beta-1)\right]-k_{1} r_{1} \mu\left(k_{2}-k_{1} \eta_{2}\right) \cosh \left[6 \sqrt{\eta_{1}} \rho(\beta-1)\right] \\
& -8 k_{1} r_{1} \sqrt{\eta_{1}} \mu \rho(\beta-1)\left(k_{2}-k_{1} \eta_{2}\right) \sinh \left[2 \sqrt{\eta_{1}} \rho(\beta-1)\right] \\
& +2 k_{1} \eta_{2}\left[k_{1} r_{1} \mu-6 \pi \eta_{1} \rho(\beta-1)\right]-4 k_{1}^{2} r_{1} \sqrt{\eta_{1}} \eta_{2} \mu \rho(\beta-1) \\
& \left.\times \sinh \left[4 \sqrt{\eta_{1}} \rho(\beta-1)\right]\right\} \\
d_{12}= & 4(\beta-1) \pi \rho \eta_{1}\left\{2\left(k_{2}^{2}-2 k_{1} k_{2} \eta_{2}+2 k_{1}^{2} \eta_{2}^{2}\right) \cosh \left[2 \sqrt{\eta_{1}} \rho(\beta-1)\right]\right. \\
& \left.-k_{1} \eta_{2}\left(k_{1} \eta_{2}-k_{2}\right)\left(3+\cosh \left[4 \sqrt{\eta_{1}} \rho(\beta-1)\right]\right)\right\}
\end{aligned}
$$

$$
\begin{aligned}
d_{13}= & 2\left(k_{1}-k_{2}\right)\left\{-\left[k_{1} r_{1} \mu-8(\beta-1) \pi \eta_{1} \rho\right] \cosh \left[2 \sqrt{\eta_{1}} \rho(\beta-1)\right]\right. \\
& +k_{1} r_{1} \mu \cosh \left[6 \sqrt{\eta_{1}} \rho(\beta-1)\right]+8 \sqrt{\eta_{1}} \rho(\beta-1) \\
& \left.\times \sinh \left[2 \sqrt{\eta_{1}} \rho(\beta-1)\right]\right\} \\
d_{14}= & \left.-2 k_{2}+k_{1}^{2} \eta_{3}+k_{1}\left(1+\eta_{2}-k_{2} \eta_{3}\right)\right]\left[k_{1} r_{1} \mu\right. \\
& \left.-16(\beta-1) \pi \eta_{1} \rho\right] \cosh \left[2 \sqrt{\eta_{1}} \rho(\beta-1)\right] \\
& +2 k_{1}\left[\eta_{2}+\left(k_{1}-k_{2}\right) \eta_{3}\right]\left[k_{1} r_{1} \mu+2(\beta-1) \pi \eta_{1} \rho\right] \\
& \times \cosh \left[4 \sqrt{\eta_{1}} \rho(\beta-1)\right]-k_{1} r_{1} \mu\left[-2 k_{2}+k_{1}^{2} \eta_{3}\right. \\
& \left.+k_{1}\left(1+\eta_{2}-k_{2} \eta_{3}\right)\right]\left\{\cosh \left[6 \sqrt{\eta_{1}} \rho(\beta-1)\right]\right. \\
& \left.+8 \sqrt{\eta_{1}} \rho(\beta-1) \sinh \left[2 \sqrt{\eta_{1}} \rho(\beta-1)\right]\right\} \\
& -2 k_{1}\left[\eta_{2}+\left(k_{1}-k_{2}\right) \eta_{3}\right]\left[-k_{1} r_{1} \mu+6 \pi \eta_{1} \rho(\beta-1)\right] \\
& -4 k_{1}^{2}\left[\eta_{2}+\left(k_{1}-k_{2}\right) \eta_{3}\right] r_{1} \sqrt{\eta_{1}} \mu \rho(\beta-1) \sinh \left[4 \sqrt{\eta_{1}} \rho(\beta-1)\right] \\
d_{15}= & 4(\beta-1) \pi \rho \eta_{1}\left\{4\left[k_{2}+k_{1} k_{2} \eta_{3}-k_{1} \eta_{2}\left(1+2 k_{1} \eta_{3}\right)\right]\right. \\
& \times \cosh \left[2 \sqrt{\eta_{1}} \rho(\beta-1)\right]+k_{1}\left(\eta_{2}-k_{2} \eta_{3}+2 k_{1} \eta_{2} \eta_{3}\right) \\
& \left.\times\left(3+\cosh \left[4 \sqrt{\eta_{1}} \rho(\beta-1)\right]\right)\right\}
\end{aligned}
$$

\section{Journal of Applied Mechanics}




$$
\begin{aligned}
d_{16}= & \left.k_{1} r_{1} \mu-8(\beta-1) \pi \eta_{1} \rho\right] \cosh \left[2 \sqrt{\eta_{1}} \rho(\beta-1)\right] \\
& -k_{1} r_{1} \mu \cosh \left[6 \sqrt{\eta_{1}} \rho(\beta-1)\right]+8 \sqrt{\eta_{1}} \rho(\beta-1) \\
& \times \sinh \left[2 \sqrt{\eta_{1}} \rho(\beta-1)\right] \\
d_{17}= & \left.-\left(1+k_{1} \eta_{3}\right)\right]\left[k_{1} r_{1} \mu-16(\beta-1) \pi \eta_{1} \rho\right] \cosh \left[2 \sqrt{\eta_{1}} \rho(\beta-1)\right] \\
& -2 k_{1} \eta_{2}\left[k_{1} r_{1} \mu+2(\beta-1) \pi \eta_{1} \rho\right] \cosh \left[4 \sqrt{\eta_{1}} \rho(\beta-1)\right] \\
& +k_{1} r_{1} \mu\left(1+k_{1} \eta_{3}\right) \cosh \left[6 \sqrt{\eta_{1}} \rho(\beta-1)\right] \\
& +8 k_{1} r_{1} \sqrt{\eta_{1}} \mu \rho(\beta-1)\left(1+k_{1} \eta_{3}\right) \sinh \left[2 \sqrt{\eta_{1}} \rho(\beta-1)\right] \\
& +2 k_{1} \eta_{3}\left[k_{1} r_{1} \mu-6 \pi \eta_{1} \rho(\beta-1)\right]-4 k_{1}^{2} r_{1} \sqrt{\eta_{1}} \eta_{2} \mu \rho(\beta-1) \\
& \times \sinh \left[4 \sqrt{\eta_{1}} \rho(\beta-1)\right] \\
d_{18}= & 4(\beta-1) \pi \rho \eta_{1}\left\{-2\left(1+2 k_{1} \eta_{3}+2 k_{1}^{2} \eta_{3}^{2}\right) \cosh \left[2 \sqrt{\eta_{1}} \rho(\beta-1)\right]\right. \\
& \left.+k_{1} \eta_{3}\left(k_{1} \eta_{3}+1\right)\left(3+\cosh \left[4 \sqrt{\eta_{1}} \rho(\beta-1)\right]\right)\right\}
\end{aligned}
$$

Combining Eqs. (A4)-(A6) yields

$$
\frac{\partial U_{f t}}{\partial \beta}=p_{1} \sigma_{0}^{2}+p_{2} \sigma_{0}+p_{3}, \quad \frac{\partial U_{m t}}{\partial \beta}=p_{4} \sigma_{0}^{2}+p_{5} \sigma_{0}+p_{6}
$$

where we have

$$
\begin{aligned}
& p_{1}=\frac{\pi r_{f 0}^{2} L}{2 E_{f}} h_{1}\left(c_{1}+c_{4}\right), \quad p_{2}=\frac{\pi r_{f 0}^{2} L}{2 E_{f}}\left[h_{1}\left(c_{2}+c_{5}\right)+h_{2}\left(c_{7}+c_{9}\right)\right] \\
& p_{3}=\frac{\pi r_{f 0}^{2} L}{2 E_{f}}\left[h_{1}\left(c_{3}+c_{6}\right)+h_{2}\left(c_{8}+c_{10}\right)\right] \\
& p_{4}=\frac{\pi r_{f 0}^{2} L}{2 E_{m}} h_{4}\left(c_{1}+c_{4}\right)+\frac{\pi r_{f 0}^{3} \gamma^{2}\left(1+\nu_{m}\right) a_{6}}{E_{m} \rho}\left(c_{11}+c_{14}\right) \\
& p_{5}=\frac{\pi r_{f 0}^{2} L}{2 E_{m}}\left[h_{4}\left(c_{2}+c_{5}\right)+h_{5}\left(c_{7}+c_{9}\right)\right]+\frac{\pi r_{f 0}^{3} \gamma^{2}\left(1+\nu_{m}\right) a_{6}}{E_{m} \rho}\left(c_{12}+c_{15}\right) \\
& p_{6}=\frac{\pi r_{f 0}^{2} L}{2 E_{m}}\left[h_{4}\left(c_{3}+c_{6}\right)+h_{5}\left(c_{8}+c_{10}\right)\right]+\frac{\pi r_{f 0}^{3} \gamma^{2}\left(1+\nu_{m}\right) a_{6}}{E_{m} \rho}\left(c_{13}+c_{16}\right)
\end{aligned}
$$

Letting $w_{1}=p_{1}+p_{4}, w_{2}=p_{2}+p_{5}, w_{3}=p_{3}+p_{6}$ leads to

$$
G_{c r} L \oint_{s_{f}} d s=\frac{\partial U_{f t}}{\partial \beta}+\frac{\partial U_{m t}}{\partial \beta}=w_{1} \sigma_{0}^{2}+w_{2} \sigma_{0}+w_{3}
$$

\section{References}

[1] Mukhopadhyay, M., 2005, Mechanics of Composite Materials and Structures, Longman, India.

[2] Gibson, R. F., 2011, Principles of Composite Material Mechanics, 3rd ed., Taylor \& Francis Group, Boca Raton, FL.

[3] Christensen, R. M., 1979, Mechanics of Composite Materials, John Wiley \& Sons, NJ.

[4] Piggort, M. R., 2002, Load Bearing Fiber Composites, 2nd ed., Kluwer, New York.

[5] Barbero, E. J., 2010, Introduction to Composite Materials Design, Taylor \& Francis Group, Boca Raton, FL.

[6] Hughes, J. D. H., 1991, "The Carbon Fiber/Epoxy Interface-A Review," Compos. Sci. Technol., 41(1), pp. 31-45.

[7] Kobets, L. P., and Deev, I. S., 1997, "Carbon Fibers: Structure and Mechanical Properties," Compos. Sci. Technol., 57(12), pp. 1571-1580.

[8] Carlson, T., Ordeus, D., Wysocki, M., and Asp, L. E., 2010, "Structural Capacitor Materials Made From Carbon Fiber Epoxy Composites," Compos. Sci. Technol., 70(7), pp. 1135-1140.

[9] Schmidt, J. F., 2011, "Methods for Manufacturing a Vehicle Comprising Carbon Fiber," U.S. Patent, 8,025,753, Sep. 272011.

[10] Li, H., Liang, H., He, F., Huang, Y., and Wan, Y. Z., 2009, “Air Dielectric Barrier Discharges Plasma Surface Treatment of Three-Dimensional Braided Carbon Fiber Reinforced Epoxy Composites," Surf. Coat. Technol., 203(10-11), pp. 1317-1321.

[11] Yan, C. Z., Hao, L., Xu, L., and Shi, Y. S., 2011, "Preparation, Characterisation and Processing of Carbon Fibre/Polyamide-12 Composites for Selective Laser Sintering," Compos. Sci. Technol., 71(16), pp. 1834-1841.
[12] Park, S. J., Kim, M. H., Lee, J. R., and Choi, S., 2000, "Effect of Fiber-Polymer Interactions on Fracture Toughness Behavior of Carbon Fiber-Reinforced Epoxy Matrix Composites,” J. Colloid Interf. Sci., 228(2), pp. 287-291.

[13] Severini, F., Formaro, L., Pegoraro, M., and Posca, L., 2002, "Chemical Modification of Carbon Fiber Surfaces," Carbon, 40(5), pp. 735-741.

[14] Chaudhuri, S. N., Chaudhuri, R. A., Benner, R. E., and Penugonda, M. S., 2006, "Raman Spectroscopy for Characterization of Interfacial Debonds Between Carbon Fibers and Polymer Matrices," Compos. Struct., 76(4), pp. 375-387.

[15] Luo, Y. F., Zhao, Y., Duan, Y. X., and Du, S. Y., 2011, "Surface and Wettability Property Analysis of CCF300 Carbon Fibers With Different Sizing or Without Sizing," Mater. Des., 32(2), pp. 941-946.

[16] Atkinson, K. E., and Kiely, C., 1998, "The Influence of Fiber Surface Properties on the Mode of Failure in Carbon-Fiber/Epoxy Composites," Compos. Sci Technol., 58(12), pp. 1917-1922.

[17] Meng, L. H., Chen, Z. W., Song, X. L., Liang, Y. X., Huang, Y. D., and Jiang, Z. X., 2009, "Influence of High Temperature and Pressure Ammonia Solution Treatment on Interfacial Behavior of Carbon Fiber/Epoxy Resin Composites,' J. Appl. Polym. Sci., 113(6), pp. 3436-3441.

[18] Fu, Y. F., Xu, K., Li, J., Sun, Z. Y., Zhang, F. Q., and Chen, D. M., 2012, “The Influence of Plasma Surface Treatment of Carbon Fibers on the Interfacial Adhesion Properties of UHMWPE Composite," Polym. Plast. Technol. Eng., 51(3), pp. 273-276.

[19] Allongue, P., Delamar, M., Desbat, B., Fagebaume, O., Hitmi, R., Pinson, J., and Saveant, J. M., 1997, "Covalent Modification of Carbon Surfaces by Aryl Radicals Generated From the Electrochemical Reduction of Diazonium Salts," J. Am. Chem. Soc., 119(1), pp. 201-207.

[20] Zhang, H., Zhang, Z., and Breidt, C., 2004, "Comparison of Short Carbon Fibre Surface Treatments on Epoxy Composites I. Enhancement of the Mechanical Properties," Compos. Sci. Technol., 64(13-14), pp. 2021-2029.

[21] Bai, Y. P., Wang, Z., and Feng, L. Q., 2010, "Interface Properties of Carbon Fiber/Epoxy Resin Composite Improved by Supercritical Water and Oxygen in Supercritical Water," Mater. Des., 31(3), pp. 1613-1616.

[22] Rhee, K. Y., Park, S. J., Hui, D., and Qiu, Y., 2011, "Effect of Oxygen PlasmaTreated Carbon Fibers on the Tribological Behavior of Oil-Absorbed Carbon/ Epoxy Woven Composites," Compos. Part B, (in press).

[23] Zhang, Z. Q., Liu, Y. W., Huang, Y. D., Liu, L., and Bao, J. W., 2002, "The Effect of Carbon-Fiber Surface Properties on the Electron-Beam Curing of Epoxy-Resin Composites," Compos. Sci. Technol., 62(3), pp. 331-337.

[24] Naves, L. Z., Santana, F. R., Castro, C. G., Valdivia, A. D., Mota, da A. S. Estrela, C., Sobrinho, C. L., and Soares, J. C., 2011, "Surface Treatment of Glass Fiber and Carbon Fiber Posts: SEM Characterization," Microsc. Res. Tech., 74(12), pp. 1088-1092.

[25] Lu, C., Chen, P., Yu, Q., Ding, Z. F., Lin, Z. W., and Li, W., 2007, "Interfacial Adhesion of Plasma-Treated Carbon Fiber/Poly(Phthalazinone Ether Sulfone Ketone) Composite," J. Appl. Polym. Sci., 106(3), pp. 1733-1741.

[26] Jiang, G., Pickering, S. J., Lester, E. H., Turner, T. A., Wong, K. H., and Warrior, N. A., 2009, "Characterization of Carbon Fibres Recycled From Carbon Fibre/Epoxy Resin Composites Using Super Critical n-Propanol," Compos. Sci. Technol., 69(2), pp. 192-198.

[27] Kim, S. Y., Baek, S. J., and Youn, J. R., 2011, "New Hybrid Method for Simultaneous Improvement of Tensile and Impact Properties of Carbon Fiber Reinforced Composites," Carbon, 49(15), pp. 5329-5338.

[28] Song, W., Gu, A. J., Liang, G. Z., and Li, Y., 2011, "Effects of Surface Roughness on Interfacial Properties of Carbon Fibers Reinforced Epoxy Resin Composites," Appl. Surf. Sci., 257(9), pp. 4069-4074.

[29] Xie, J. F., Xin, D. W., Cao, H. Y., Wang, C. T., Zhao, Y., Yao, L., Ji, F., and Qiu, Y. P., 2011, "Improving Carbon Fiber Adhesion to Polyimide With Atmospheric Pressure Plasma Treatment," Surf. Coat. Technol., 206(2-3), pp. 191-201.

[30] Kerans, R. G., and Parthasarathy, T. A., 1991, "Theoretical Analysis of the Fiber Pullout and Pushout Tests," J. Am. Ceram. Soc., 74(7), pp. 1585-1596.

[31] Liu, H. Y., Zhou, L. M., and Mai, Y. W., 1994, "On Fiber Pull-Out With a Rough Interface," Philos. Mag. A, 70(2), pp. 359-372.

[32] Parthasarathy, T. A., Marshall, D. B., and Kerans, R. G., 1994, "Analysis of the Effect of Interfacial Roughness on Fiber Debonding and Sliding in Brittle Matrix Composites," Acta Metall. Mater., 42(11), pp. 3773-3784.

[33] Chai, Y. S., and Mai, Y. W., 2001, "New Analysis on the Fiber Push-Out Problem With Interface Roughness and Thermal Residual Stresses," J. Mater. Sci. 36(8), pp. 2095-2104.

[34] Jiang, L. Y., Huang, Y., Jiang, H., Ravichandran, G., Gao, H. J., Hwang, K. C., and Liu, B., 2006, "A Cohesive Law for Carbon Nanotube/Polymer Interfaces Based on the Van der Waals Force," J. Mech. Phys. Solids, 54(11), pp. 2436-2452.

[35] Waters, J. F., Lee, S., and Guduru, P. R., 2009, "Mechanics of Axisymmetric Wavy Surface Adhesion: JKR-DMT Transition Solution," Int. J. Solids Struct., 46(5), pp. 1033-1042.

[36] Hutchinson, J. W., and Jensen, H. M., 1990, "Models of Fiber Debonding and Pullout in Brittle Composites With Friction," Mech. Mater., 9(2), pp. 139-163.

[37] Gao, Y. C., Mai, Y. W., and Cotterell, B., 1988, "Fracture of Fiber-Reinforced Materials," J. Appl. Math. Phys., 39(4), pp. 550-572.

[38] Fu, S. Y., Yue, C. Y., Hu, X., and Mai, Y. W., 2000, "Analyses of the Micromechanics of Stress Transfer in Single- and Multi-Fiber Pull-Out Tests," Compos. Sci. Technol., 60(4), pp. 569-579.

[39] Whitney, J. M., and Riley, M. B., 1966, "Elastic Properties of Fiber Reinforced Composite Materials," AIAA. J., 4(9), pp. 1537-1542.

[40] Zhang, B. M., Yang, Z., and Sun, X. Y., 2010, "Measurement and Analysis of Residual Stresses in Single Fiber Composite," Mater. Des., 31(3), pp. $1237-1241$. 
[41] Brandstetter, J., Kromp, K., Peterlik, H., and Weiss, R., 2005, "Effect of Surface Roughness on Friction in Fiber-Bundle Pull-Out Tests," Compos. Sci. Technol., 65(6), pp. 981-988.

[42] Cox, H. L., 1952, "The Elasticity and Strength of Paper and Other Fibrous Materials," Br. J. Appl. Phys., 3(3), pp. 72-79.

[43] Lin, Z., and Li, C. V., 1997, "Crack Bridging in Fiber Reinforced Cementitious Composites With Slip-Hardening Interfaces," J. Mech. Phys. Solids, 45(5), pp 763-787.

[44] Mackin, T. J., Warren, P. D., and Evans, A. G., 1992, "Effects of Fiber Roughness on Interface Sliding in Composites," Acta Metall. Mater., 40(6), pp 1251-1257.

[45] Hampe, A., Kalinka, G., Meretz, S., and Schulz, E., 1995, "An Advanced Equipment for Single-Fibre Pullout Test Designed to Monitor the Fracture Process," Compos., 26(1), pp. 40-46.

[46] Francia, C. D., Ward, T. C., and Claus, R. O., 1996, "The Single-Fibre Pullout Test. 1: Review and Interpretation," Compos. Part A, 27(8), pp 597-612.

[47] Huang, Y. L., and Young, R. J., 1996, "Interfacial Micromechanics in Thermoplastic and Thermosetting Matrix Carbon Fiber Composites," Compos. Part A, 27(10), pp. 973-980.
[48] Piggott, M. R., 1991, "Failure Processes in the Fibre-Polymer Interphase," Compos. Sci. Technol., 42(1-3), pp. 57-76.

[49] Bismarck, A., Menner, A., Kumru, M. E., Sarac, S. A., Bistritz, M., and Schulz, E., 2002, "Poly(Carbazole-co-Acrylamide) Electrocoated Carbon Fibers and Their Adhesion Behavior to an Epoxy Resin Matrix," J. Mater. Sci., 37(3), pp. 461-471.

[50] Tsai, K. H., and Kim, K. S., 1996, "The Micromechanics of Fiber Pullout," J. Mech. Phys. Solids, 44(7), pp. 1147-1177.

[51] Yue, C. Y., and Cheung, W. L., 1992, "Interfacial Properties of FiberReinforced Composites,” J. Mater. Sci., 27(14), pp. 3843-3855.

[52] Liu, Y. F., and Kagawa, Y., 1996, "Analysis of Debonding and Frictional Sliding in Fiber-Reinforced Brittle Matrix Composites: Basic Problems," Mater. Sci. Eng. A, 212(1), pp. 75-86.

[53] Marshall, D. B., Cox, B. N., and Evans, A. G., 1985, "The Mechanics of Matrix Cracking in Brittle-Matrix Fiber Composites," Acta Metall., 33(11), pp. 2013-2021.

[54] Hsueh, C. H., 1995, "Matrix Cracking With Frictional Bridging Fibers in Continuous Fiber Ceramic Composites," J. Mater. Sci., 30(7), pp. 1781-1789.

[55] Evans, A. G., and Marshall, D. B., 1989, "The Mechanical Behavior of Ceramic Matrix Composites,” Acta Metall., 37(10), pp. 2567-2583. 